\title{
Optimal sales and production rollover strategies under capacity constraints
}

\author{
Justus Arne Schwarz ${ }^{\mathrm{a}, *}$, Barış Tan ${ }^{\mathrm{b}}$ \\ ${ }^{a}$ Chair of Production Management, University of Mannheim, Germany \\ ${ }^{\mathrm{b}}$ College of Administrative Sciences and Economics, Koç University, Istanbul, Turkey
}

\section{A R T I C L E I N F O}

\section{Article history:}

Received 4 August 2020

Accepted 25 January 2021

Available online $\mathrm{xxx}$

\section{Keywords:}

Production

Product rollover

Capacity

New product introduction

\begin{abstract}
A B S T R A C T
Firms regularly replace their old product generation by a newer generation to sustain and increase their market share and profit. The product rollover problem of deciding on the number of old products to be pre-produced before the introduction of the new generation, and then deciding on the prices, sales volumes, and production volumes of the old and the new generation during the introduction under capacity constraint is considered.

Production capacity limitations are common during the introduction period of a new product. We provide the first study that examines how a production capacity constraint affects the optimal decisions. The optimal decisions for a deterministic period-based model are provided in closed-form.

A single sales/production rollover strategy implies that the sales/production of the old generation is discontinued before introducing the new generation. With a dual sales/production rollover strategy, the old and the new generation are sold/produced simultaneously. Depending on the capacity shortage, there are two types of mitigation actions: (i) increasing the prices, (ii) changing the sales and/or production rollover strategies with pre-production while adjusting the prices accordingly. If the capacity is unlimited, aligned sales and production rollover strategies are always optimal. We establish the conditions under which limited capacity leads to a combination of a single production rollover with a dual sales rollover strategy. We show that the selection of optimal rollover strategies is non-monotone in the available capacity. This implies that a change in the rollover strategy in response to limiting capacity has to be revoked for more severe capacity shortages.
\end{abstract}

(c) 2021 Elsevier B.V. All rights reserved.

\section{Introduction}

In many industries, such as home appliances, consumer electronics, and semiconductors industry, firms regularly replace their old product generation by a newer generation to sustain and increase market share and profit. Introducing a new product generation may also require a controlled phase-out of the old product from the market and the manufacturing system.

There are two strategies for offering new and old products to customers: a single rollover or a dual rollover (Billington, Lee, \& Tang, 1998). A dual rollover refers to a transition in which both generations are sold simultaneously. This strategy leaves the firm exposed to cannibalization effects between the generations and postponement of purchasing decisions by strategic customers. By choosing a single rollover strategy, i.e., removing the old generation

\footnotetext{
* Corresponding author.

E-mail address: schwarz@bwl.uni-mannheim.de (J. A. Schwarz).
}

from the market prior to the introduction of the new generation, both negative effects can be avoided. However, this comes at the expense of lost revenue from the old generation.

This paper investigates the case of a new product introduction in the presence of a previous generation and finite production capacity. In order to differentiate the two market transition strategies from the transition taking place on the shop floor, we introduce the terms sales rollover and production rollover. We use the terms single sales rollover (SSR) and dual sales rollover (DSR) to refer to the two market transition strategies described above. Analogously, a dual production rollover (DPR) refers to simultaneous production of both products. A single production rollover (SPR) refers to stopping the production of the old generation before the ramp-up of the new generation.

The advantage of a DPR is that it aligns production with the sales of a DSR without pre-production. However, it requires either building up a dedicated production capacity for the new generation or flexible capacity that is shared between both generations. Shared capacity is common for assembly processes found e.g. at 
home appliance and automotive manufacturers (Clark \& Fujimoto, 1991), and in industries where technological upgrades result in flexible capacity such as the semiconductor industry (Bansal, Uzsoy, \& Kempf, 2020; Li, Graves, \& Huh, 2014). In the case of limited capacity, the disadvantage of the DPR is that the new and the old generations compete for the shared capacity. An SPR, on the contrary, frees up the capacity for the new generation. However, it requires building up inventory of the old product if a DSR is planned.

In this paper, we analyze the optimal sales and production rollover strategies and the underlying optimal prices, sales volume, and production volume in the presence of a finite production capacity to answer the following research questions. (i) What are the optimal sales and production rollover strategies to mitigate the negative effects of limited capacity? (ii) How much does a firm lose if it ignores capacity limitations when deciding its sales rollover strategy and prices?

To answer these research questions, we introduce a model for a profit-maximizing monopolist that decides the sales and production rollover strategies and the production, sales, and prices for both generations in a deterministic setting. While the new generation with improved product quality can only be produced after the start of the introduction period, the firm has the option to pre-produce old products at the expense of additional inventory holding costs. The old and the new generation are produced with a shared and finite production capacity. The capacity consumption of the new generation and its variable production cost may be different from the old generation. Customers base their purchasing decisions on prices in relation to the perceived quality of the offered product generations.

For example, home appliances and white goods manufacturers face this problem of selecting sales and production rollover strategies on a regular basis, since they introduce new models of washing machines and dryers approximately every two to three years. The home appliances market typically features gradual improvements. In particular, increased energy efficiency is one aspect that improves from one model generation to the next (Michel, Attali, \& Bush, 2016). The ramp-up of the production of a new model typically takes three to six months. During the ramp-up, the production output has not yet reached its target level due to technical difficulties with new processes and shortages of critical components. Manufacturers may produce the old and the new generation on the same assembly line to meet the demand with their limited capacity.

Despite the importance of limited production capacities during the introduction of a new generation, analytical models that have analyzed optimal rollover strategies so far have assumed unlimited capacities. We contribute to the literature by presenting the optimal solution of the firm's optimization problem analytically and by characterizing its structure. Moreover, we derive the following insights regarding the impact of a limited production capacity on optimal sales and production rollover strategies.

Depending on the severity of the capacity limitation, different actions can be taken to mitigate the effects of limited capacity: (i) price increases, and (ii) changes in sales and/or production rollover strategies and exploitation of the pre-production of the old product. A differentiation between sales and production rollover strategies is required because in contrast to the unlimited capacity case, optimal sales and production rollover strategies are not necessarily aligned if the capacity is limited. Limited production capacity is one of the drivers behind firms selecting a DSR instead of an SSR strategy because it allows for a DPR strategy where the production can be shifted to a less capacity-demanding older generation. Moreover, a DSR allows for the exploitation of the pre-production of the old product.

One might expect that if the rollover strategy is changed in response to a limited capacity this change is optimal for all set- tings in which the capacity is even more restrictive. However, we find that for new product generations with a medium quality increase, the selection of optimal rollover strategies is non-monotone in the available capacity. This is due to the substitution effects between the product generations and implies that a change in the rollover strategy in response to limiting capacity has to be revoked for more severe capacity shortages.

Finally, our results show that integrated sales and operations planning provides substantial improvements over a sequential planning approach. The sequential planning approach neglects capacity limitations in the first step when selecting the sales rollover strategy and only considers capacity restrictions when determining the actual production to comply with the previously selected sales rollover strategy.

The remainder of the paper is organized as follows. We begin with a review of the related literature in Section 2. Section 3 introduces the model and its underlying assumptions. The optimal decisions are analyzed in Section 4. The sensitivity of the optimal strategies in key model parameters is discussed in Section 5. Moreover, the proposed integrated sales and operations planning is compared numerically with a sequential planning approach. Section 6 summarizes the obtained managerial insights. Concluding remarks and potential areas of future research are presented in Section 7.

\section{Literature review}

Our work is related to the literature on sales and operations planning for new product introductions. In contrast to classical models of competing products with different qualities, such as discussed in Moorthy (1988) and Mussa and Rosen (1978), product rollover models need to cover the dynamics of product availability and other operational requirements during the introduction of a new product generation. There are studies on new product introductions with a limited production capacity but without preceding product generations, e.g., Jain, Mahajan, and Muller (1991), Kumar and Swaminathan (2003), Ho, Savin, and Terwiesch (2002), Ho, Savin, and Terwiesch (2011), Shen, Duenyas, and Kapuscinski (2011), Shen, Duenyas, and Kapuscinski (2014), and Bilginer and Erhun (2015). Due to the absence of a predecessor generation, these studies do not discuss rollover strategies. Our study focuses on the introduction of new products in the presence of predecessor generations such that a rollover strategy has to be selected.

For product introductions with predecessors, Billington et al. (1998) relate the optimal selection of strategies to the firms' risk profiles based on case studies. They recommend the DSR strategy for firms exposed to high market and product risks and the SSR strategy in low-risk situations. Erhun, Gonçalves, and Hopman (2007) describe a case where Intel Corp. struggled with pricing and limited production capacities during the transition to a new microprocessor generation.

In contrast to a product rollover, a product line extension means that the old version is complemented by a new product version and both versions will remain in the market in the long run (see e.g. Wilson \& Norton, 1989). The existing analytical models for product rollovers have two different foci. They either analyze the optimal selection of rollover strategies but do not consider operational aspects such as a limited production capacity or they consider the operational aspects but assume fixed rollover strategies.

The studies that analytically analyze the optimal selection of rollover strategies focus on sales rollover strategies. Whether an SSR or a DSR strategy is recommended depends on the assumptions regarding the market setting and the customer choice model. Features considered in these studies include the availability of a second hand-market for durable goods (Levinthal \& Purohit, 1989), the development of demand over time (Lim \& Tang, 2006), the use 
of a demand function that depends on the diffusion of awareness (Koca, Souza, \& Druehl, 2010), the consideration of a decrease in the perceived value of the new product if the customers already have bought the old product (Zhou, Zhang, Gou, \& Liang, 2015), the presence of leftovers of the old generation due to random demand (Ferguson \& Koenigsberg, 2007), and the modeling of demand uncertainty during the transition period (Li \& Graves, 2012). In addition, if customers are capable of acting strategically, they consider the expected rollover strategy of a firm when making a decision about the buying or delaying of their purchase (Bernstein \& Martínez-de-Albéniz, 2017; Liang, Çakanyıldırım, \& Sethi, 2014; Liu, Zhai, \& Chen, 2018; 2019).

The common modeling approach is to capture the dynamics of a product rollover in a two-period model with a focus on the substitution effects between the product generations. All of these twoperiod models assume that the relevant information is deterministic during the second period in which a new generation is potentially introduced. Ferguson and Koenigsberg (2007) and Liang et al. (2014) consider stochastic demand for the old product in the first period while the remaining models are completely deterministic.

In general, these models assume that the production rollover strategy is aligned with the sales rollover strategy. However, Koca et al. (2010), Ferguson and Koenigsberg (2007), and Liang et al. (2014) assume a fixed SPR for technological reasons or because sales of the old generation occur only because of leftovers caused by random demand. The firms never produce the old generation with inferior quality on purpose.

Even though the need for considering a finite production capacity is acknowledged, among others by Koca et al. (2010), all of the references reviewed above assume unlimited production capacities.

The existing studies that consider finite capacity in new product introduction either explicitly assume a given sales and production rollover strategy (Sale, Mesak, \& Inman, 2017), the optimal strategy is implicitly given by the model assumptions (Bansal et al., 2020; Li et al., 2014), or do not consider pricing (Negahban \& Smith, 2018). Except for Sale et al. (2017), prices are given exogenously and do not affect customer behavior. All models are deterministic with the exception of Li et al. (2014) who consider random demand.

Sale et al. (2017) model the diffusion of awareness with a Bass diffusion model and decide on the pricing, lot sizes, and the length of a sales period while considering inventory holding costs, setup costs, and fixed costs to introduce a new generation. Their model assumes a given SSR and SPR strategy. Li et al. (2014) focus on finding an optimal conversion and purchasing plan for the capacity. The demand for both generations is given exogenously and has to be fulfilled according to a service level agreement. Therefore, all of the considered cases feature a DSR and a DPR strategy. Negahban and Smith (2018) consider timing, capacity, sales and production decisions in the introduction of two successive generations with a capacity constraint for the new generation. This model assumes independent capacities for both products and includes neither the strategic customers nor the cannibalization of sales of the new generation by the old. Bansal et al. (2020) develop a coordination mechanism to share capacity between existing products, test runs for new product generations, and production of new product generations. The demand for the different product generations is given exogenously.

To the best of our knowledge, our model is the first to analyze the optimal selection of sales and production rollover strategies with the corresponding optimal pricing decisions while considering limited production capacities.

\section{Model}

This section presents the assumptions regarding the customer behavior, the operational setting of the firm, and the resulting op-
Table 1

Model notation.

\begin{tabular}{|c|c|}
\hline \multicolumn{2}{|l|}{ Indices } \\
\hline$o, n$ & product generations old $(0)$ and new $(n)$ \\
\hline \multicolumn{2}{|c|}{ Parameters } \\
\hline$\tau^{0}$ & length of the of pre-introduction period \\
\hline$\tau$ & length of the introduction period \\
\hline$c$ & $\begin{array}{l}\text { variable production costs for producing one unit of the old } \\
\text { generation }\end{array}$ \\
\hline$\alpha$ & $\begin{array}{l}\text { relative difference in variable production costs of the new compared } \\
\text { to the old generation }\end{array}$ \\
\hline$h$ & holding costs for pre-produced old products \\
\hline$\Delta$ & $\begin{array}{l}\text { relative difference in the quality of the new generation compared to } \\
\text { the old generation }\end{array}$ \\
\hline$\theta$ & quality valuation parameter \\
\hline$k^{0, t o t a l}$ & total capacity during the pre-introduction period \\
\hline$k^{0}$ & $\begin{array}{l}\text { capacity to pre-produce the old generation during the } \\
\text { pre-introduction period }\end{array}$ \\
\hline$k$ & capacity during the introduction period (in old product units) \\
\hline$\beta$ & $\begin{array}{l}\text { relative difference in capacity consumption of the new compared to } \\
\text { the old generation }\end{array}$ \\
\hline$x^{0,0, *}$ & $\begin{array}{l}\text { production of the old generation for sales in the pre-introduction } \\
\text { period }\end{array}$ \\
\hline \multicolumn{2}{|c|}{ Decision variables } \\
\hline$P^{o}, P^{n}$ & price of the old and the new generation in the introduction period \\
\hline$X^{o}, X^{n}$ & $\begin{array}{l}\text { production of the old and the new generation in the introduction } \\
\text { period }\end{array}$ \\
\hline$X_{\text {pre }}^{o}$ & $\begin{array}{l}\text { pre-production of the old the generation in the pre-introduction } \\
\text { period }\end{array}$ \\
\hline$S^{o}, S^{n}$ & sales of the old and the new generation in the introduction period \\
\hline
\end{tabular}

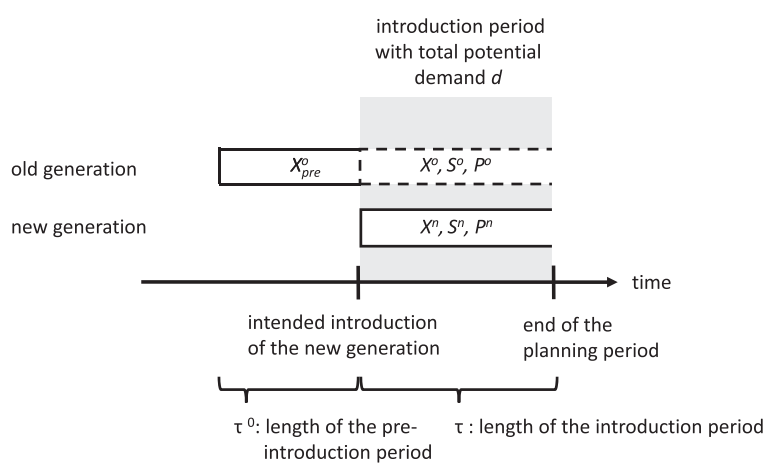

Fig. 1. Period based model of the transition period with production $X_{p r e}^{o}, X^{o}, X^{n}$, sales $S^{o}, S^{n}$, and prices $P^{o}, P^{n}$.

timization problem for the firm. The notation is summarized in Table 1 . The model aggregates the continuous time in two periods to capture the relevant dynamics. These periods are a preintroduction period and the introduction period for which the new product introduction is intended (Fig. 1). The timing of the introduction and the length of the pre-introduction period $\tau^{0}$ and the length of the introduction period $\tau$ are exogenously given. Hence, the decisions on production quantities and sales quantities are made for cumulative values during the pre-introduction period and the introduction period. Capacity restrictions and holding costs are defined accordingly.

The following numerical example illustrates the decisions of a white-goods appliances manufacturer that considers introducing a new product generation on February 1st and phasing out the old generation after a six month introduction period $(\tau)$ by August 1 st. The firm uses the last month $\left(\tau_{0}\right)$ before the introduction to build up inventory to be sold during the introduction period. The firm may choose a DSR and sell $S^{0}=100$ units of the old generation at $P^{0}=\$ 300$ and $S^{n}=500$ new products at $P^{n}=\$ 400$ during the introduction period. The realized sales of the firm depend on the 
customer's buying decisions. Customers of home appliances mainly focus on the price and key features such as energy efficiency and the capacity of the white good when making their purchasing decision (Policy Studies Institute, 2006). By the time the customers enter the market, the product in their possession is typically outdated because the lifetime of a home appliance of eight to ten years exceeds several new product introduction cycles (Wieser, Tröger, \& Hübner, 2015). A survey indicated that $88 \%$ of customers in the US enter the market in home appliances because their old product broke down (Statista, 2017). The absence of a working product at home limits the ability of these customers to postpone a purchasing decision for strategic reasons. Hence, the customers that consider buying during the pre-introduction period and the introduction period can be considered to be independent. A feasible production plan to support the DSR is the production of $X^{n}=500$ new products and $X^{0}=50$ old products during the introduction period in addition to $X_{\text {pre }}^{o}=50$ pre-produced old products. The objective of the firm is to maximize its profit by finding the optimal values of production quantities $X_{\text {pre }}^{o}, X^{o}, X^{n}$, sales quantities $S^{o}, S^{n}$ and prices $P^{0}, P^{n}$. The firm also has to optimize the production $x^{o, 0, *}$, price, and sales of the old product during the pre-production period. The model captures the result of these decisions by a limited ability of the firm to pre-produce (see Section 3.2).

\subsection{Demand model}

We assume that the firm is a monopolist that faces an exogenously given and deterministic potential demand. The potential demand refers to the maximum number of new and old units that can be sold in the market. Due to customer preferences and pricing, only a portion of the potential demand can be converted into actual sales. During the introduction period, there is a potential demand for $d$ products. We further assume that $d$ is large enough to be treated as continuous. The potential demands of the preintroduction period and the introduction period are assumed to be independent. This assumption is suitable for products whose purchases can hardly be postponed and have product lifetimes that exceed multiple introduction cycles, e.g., home appliances. The customers choose between the old and the new product generations that are offered by the firm in the introduction period. A vertical demand model captures the heterogenous willingness to pay of different customers, the substitution effects between the two product generations, and the price sensitivity of customers. This modeling approach is common in the literature (e.g., Ferguson \& Koenigsberg, 2007; Zhou et al., 2015). The old and the new generation have given quality levels $q>0$ and $q(1+\Delta)>0$, respectively. The parameter $\Delta>0$ captures the relative increase in the quality level, e.g., it is determined by the set of new features or the improved energy efficiency.

The customers decide on buying the old generation, buying the new generation, or not buying at all, based on their net utility. The net utilities $u^{o}(\theta)$ and $u^{n}(\theta)$ are given for the old and the new generation by

$u^{o}(\theta)=q \theta-P^{o}, u^{n}(\theta)=q(1+\Delta) \theta-P^{n}$,

where $P^{o}$ and $P^{n}$ represent the prices for the old and the new product. The valuation of the quality level is modeled by the factor $\theta$. A customer with a high value of $\theta$ cares more about the quality level, whereas a customer with a small $\theta$ is more concerned about the price. The customers are heterogeneous in their valuation of the product quality. We assume that $\theta$ is uniformly distributed between 0 and 1 (see, e.g., Zhou et al., 2015). Each customer will buy the available product generation that provides the highest non-negative net utility. We define $\theta^{\prime}=\frac{P^{n}-P^{0}}{q \Delta}$ as the quality valuation for which a customer is indifferent between buying the old and the new product generation. From the net utilities,

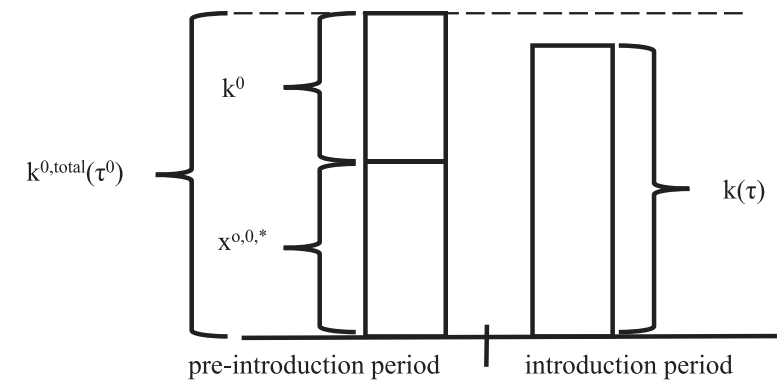

Fig. 2. Available capacity during pre-introduction period and introduction period.

we derive the demand for the old and the new product generations that limit the sales according to (2f) and (2g), depending on the sales rollover strategy. The details of the derivation, in which we normalize the demand potential to $d=1$ and the quality level $q=1$, can be found in Appendix A. In this model, the potential effect of stock-out based substitution is ignored.

\subsection{Operational setting}

The firm decides on the quantities of the old and the new product generation $X^{0}$ and $X^{n}$ to be produced during the introduction period. The variable production costs of the old and the new generation, $c>0$ and $c(1+\alpha)>0$, can be different. The parameter $\alpha>$ -1 represents the relative difference between the production costs of the new and the old generation. Positive values of $\alpha$ capture the effects of the increased costs of raw materials or components for the new generation. We exclude trivial cases where there exists no price that ensures both a positive net utility for customers and a positive profit margin for the firm exist. For the old product a price $P^{o}>c$ is required for a positive margin and $P^{0}<q=1$ is required for a positive net utility of the customers. Thus, the analysis focuses on cases with $1>c$. Respectively, for the new product $P^{n}>c(1+\alpha)$ and $P^{n}<(1+\Delta) q=1+\Delta$, i.e. $(1+\Delta)>c(1+\alpha)$ is required.

The production capacity of the firm is finite during both periods. Fig. 2 illustrates that for a given production rate, e.g., per shift, the capacity $k^{0, t o t a l}$ and $k$ are functions of the length of the preintroduction period $\tau^{0}$ and the length of the introduction period $\tau$, respectively. Hence, $k^{0, t o t a l}$ and $k$ may differ even if the production rate remains constant.

We assume that the production capacity $k$ during the introduction period is shared between the product generations. Shared capacity occurs when the two product generations are produced with the same resource, e.g., on the same assembly line. The capacity consumption per produced unit can be different for the two product generations, and their relative difference is given by $\beta$. If the entire capacity is devoted exclusively to one generation, $k$ units of the old generation or $\frac{k}{1+\beta}$ units of the new generation can be produced. Higher or equal capacity consumption $\beta \geq 0$ for the new product, i.e., $\beta>0$, is often observed in the introduction period because of learning effects still to occur or lower yield of the new product. Even if new designs and new manufacturing technologies are expected to decrease capacity consumption in the long run, the new environment may lead to an increased capacity consumption during the production ramp up in the introduction period. The analysis of the case $\beta<0$ has been conducted but is omitted in this paper.

The new generation is produced during the introduction period. However, the firm may also decide to pre-produce $X_{\text {pre }}^{o}$ units of the old generation during the pre-introduction period. Inventory holding costs of $0 \leq h<1-c$ occur per pre-produced unit. We focus on $h<1-c$ because otherwise pre-production is never profitable. The 
total production capacity during the pre-introduction period $k^{0, t o t a l}$ can be used to produce old products $x^{0,0, *}$ which are directly sold and for pre-production $X_{\text {pre }}^{o}$. Without loss of generality, the model focuses on the remaining capacity $k^{0}=\max \left\{k^{0, \text { total }}-x^{0,0, *}, 0\right\}$ after optimal production decisions $x^{0,0, *}$ for sales during the preintroduction period are made. This simplification of the model can be made as it is always optimal to prioritize sales of the old product in the pre-introduction period over future sales during the introduction period. This is due to avoidable inventory holding costs and a potentially lower price during the introduction period in response to cannibalization by the new product generation. Cases in which the sales during the pre-introduction period are limited by the capacity $k^{0, t o t a l}$ are captured by setting the remaining capacity for pre-production $k^{0}=0$.

\subsection{The firm's optimization problem}

The firm's optimization problem is given by (2). The objective of the firm is to maximize the profit $\pi$ from the revenue of units sold of the old $S^{0}$ and of the new generation $S^{n}$, while taking into account the costs of production and pre-production (Eq. (2a)). The decisions regarding prices, the number of units to be sold, and the number of units to be produced are subject to the following constraints: the firm can only sell old products that are either pre-produced or produced during the introduction period (Constraint (2b)), and the new products have to be produced during the introduction period (Constraint (2c)). As the demand is deterministic, overproduction will never occur and both constraints have to be fulfilled with equality. The pre-production must not exceed $k^{0}$ (Constraint $(2 \mathrm{~d})$ ), and the production during the introduction period must not exceed the available capacity $k$ that is shared between both product generations (Constraint (2e)). The amount of the old and the new generation that can be sold is limited by the demand function that depends on the sales rollover strategy, the prices, and the difference in quality levels (Constraints (2f) and (2g)). Finally, prices, sales, and production quantities are non-negative (Constraint $(2 \mathrm{~h})$ )

$\max \pi=P^{n} S^{n}-c(1+\alpha) X^{n}+P^{o} S^{o}-c X^{o}-(h+c) X_{\text {pre }}^{o}$

s.t.

$S^{o}=X_{\text {pre }}^{o}+X^{o}$,

$S^{n}=X^{n}$,

$k^{0} \geq X_{\text {pre }}^{o}$,

$k \geq X^{0}+(1+\beta) X^{n}$,

$S^{o} \leq \begin{cases}\min \left\{1 ; \theta^{\prime}\right\}-\min \left\{1 ; P^{o} ; \theta^{\prime}\right\} & \text { if } S^{n}>0, \\ 1-\min \left\{1 ; P^{o}\right\} & \text { otherwise }\end{cases}$

$S^{n} \leq \begin{cases}1-\min \left\{1 ; \max \left\{\frac{P^{n}}{1+\Delta} ; \theta^{\prime}\right\}\right\} & \text { if } S^{o}>0, \\ 1-\min \left\{\frac{P^{n}}{1+\Delta} ; 1\right\} & \text { otherwise, }\end{cases}$

$P^{o}, P^{n}, S^{o}, S^{n}, X^{o}, X^{n}, X_{\text {pre }}^{o} \geq 0$.
The sales and production rollover strategies are determined by the sales and production decisions. A solution where only the new generation is sold $\left(S^{o}=0, S^{n}>0\right)$ corresponds to an SSR strategy. On the other hand, the solution where both generations are sold $\left(S^{o}>0, S^{n}>0\right)$ corresponds to a DSR strategy. Analogously, producing only the new generation $\left(X^{0}=0, X^{n}>0\right)$ implies an SPR strategy whereas producing both generations $\left(X^{0}>0, X^{n}>0\right)$ corresponds to a DPR strategy. The model also allows for $X^{n}=S^{n}=0$ that corresponds to a "do not introduce" the new product strategy. The model thereby captures situations in which it is not profitable to introduce the new generation at all.

\section{Analysis of the model}

In the following, we present the analytical solution to the firm's optimization Problem (2). The solution to the optimization problem (2), is given in closed form by Theorem 1 . The proof of Theorem 1 and all further proofs can be found in Appendix B.

Theorem 1. For a firm that optimizes Problem (2), the optimal sales and production rollover strategies as well as the respective optimal pricing, production, and sales decisions for the old generation $P^{o, *}, X^{o, *}, X_{\text {pre }}^{0,}, S^{o, *}$, the new generation $P^{n, *}, X^{n, *}, S^{n, *}$, and the resulting profit $\pi^{*}$ are given

- in Table 2 if $k^{0} \geq w$ (Constraint (2d) is not binding)

- in Table 3 if $0 \leq k^{0}<w$ (Constraint (2d) binding)

where

$w= \begin{cases}\frac{1-c-h}{2}-\frac{k}{1+\beta} & \left(k<\tilde{k}_{3} \wedge \tilde{\Delta}_{3} \leq \Delta<\tilde{\Delta}_{4}\right) \\ & \vee\left(k<\tilde{k}_{1} \wedge \tilde{\Delta}_{4} \leq \Delta\right), \\ \frac{\Delta(1+\beta)-c(\Delta+\alpha \beta)-h\left(\Delta+\beta^{2}\right)}{2 \Delta}-k & \tilde{k}_{3} \leq k<\tilde{k}_{2} \wedge \tilde{\Delta}_{3}<\Delta<\tilde{\Delta}_{4}, \\ \frac{1-c-h}{2}-k & k<\tilde{k}_{4} \wedge \Delta \leq \tilde{\Delta}_{3}\end{cases}$

and $\bar{k}_{1}=\frac{(1+\beta)(1+\Delta-c(1+\alpha))}{2(1+\Delta)}, \bar{k}_{2}=\frac{(1+\beta) \Delta-c(\alpha \beta+\Delta)}{2 \Delta}$,

$\bar{k}_{3}=\frac{1-c}{2}, \tilde{k}_{1}=\frac{(1+\beta)(1-c-h)}{2}, \tilde{k}_{2}=\frac{\Delta(1+\beta)-c(\alpha \beta+\Delta)-h\left(\Delta+\beta^{2}\right)}{2 \Delta}$,

$\tilde{k}_{3}=\frac{(1+\beta)(\Delta-\beta h-\alpha c)}{2 \Delta}, \tilde{k}_{4}=\frac{1-c-h}{2}, \hat{k}_{1}=\left(1+\frac{c(\beta-\alpha)}{(\Delta-\beta)}\right) \frac{(1+\beta)}{2}$,

$\hat{k}_{2}=\frac{\beta-\Delta-c(\beta-\alpha)}{2 \beta}, \tilde{\Delta}_{1}=\alpha, \tilde{\Delta}_{2}=c \alpha, \tilde{\Delta}_{3}=h \beta+c \alpha, \tilde{\Delta}_{4}=\frac{h \beta+c \alpha}{c+h}$,

$x_{\text {pre }, \bar{k}}^{o}=\frac{-\beta^{2}(c-1)-\beta(c+\Delta+2 k-1)+\Delta(2 k-1)+\alpha(c(1+\beta))}{2 \beta(1+\beta)}$, and

$x_{p r e, \underline{k}}^{0}=\frac{\beta-\Delta-c(\beta-\alpha)}{2 \beta}-k$.

Theorem 1 shows that there are twelve regions $(A, B, \ldots, L)$ in which the optimal decisions differ structurally (see Table 2 for sufficient pre-production capacity $k^{0}$ and Table 3 if the capacity to pre-produce is binding). The optimal decisions and the thresholds $\left(\bar{k}_{1}, \bar{k}_{2} \ldots, X_{\text {pre }, \bar{k}}^{o}\right)$ that separate the regions depend on the available capacity, inventory holding costs, and variable production costs, as well as the relative difference in variable costs, capacity consumption, and quality.

Theorem 1 shows that for parameter combinations that fall into the regions $(A),(D)$, and $(G)$ the optimal decisions do not depend on the capacity $k$ during the introduction period. Hence, we refer to these regions as cases with sufficient capacity. We refer to parameter combinations as minor capacity shortages if they fall in regions that are bordered from above in terms of capacity $k$ by a region with sufficient capacity. This applies to certain parameter combinations in regions $(B),(E)$, and $(H)$. In these cases, the optimal decisions depend on the capacity during the introduction period $k$. We term parameter configurations as cases of severe capacity shortages if the optimal decisions depend on the capacity $k$ but they are bounded from above by a region with minor capacity shortages. This is the case for all parameter configurations in regions $(C),(F),(I),(J),(K)$, and $(L)$. 
Table 2

Firm's optimal decisions and resulting profit if capacity to pre-produce is sufficient.

\begin{tabular}{|c|c|c|c|}
\hline & $(A): \bar{k}_{1} \leq k \wedge \tilde{\Delta}_{1} \leq \Delta$ & $\begin{array}{l}(B):\left(\tilde{k}_{1} \leq k<\bar{k}_{1}\right) \wedge\left(\left(k \leq \hat{k}_{1} \wedge \Delta>\beta\right)\right. \\
\left.\vee\left(k \geq \hat{k}_{1} \wedge \Delta<\beta\right) \vee\left(\Delta \geq \tilde{\Delta}_{1} \wedge \alpha=\beta\right)\right)\end{array}$ & $(C):\left(k<\tilde{k}_{3} \wedge \tilde{\Delta}_{3} \leq \Delta<\tilde{\Delta}_{4}\right) \vee\left(k<\tilde{k}_{1} \wedge \tilde{\Delta}_{4} \leq \Delta\right)$ \\
\hline Rollover strategy & $\begin{array}{l}\text { single sales rollover, single } \\
\text { production rollover }\end{array}$ & $\begin{array}{l}\text { single sales rollover, single production } \\
\text { rollover }\end{array}$ & dual sales rollover, single production rollover \\
\hline $\begin{array}{l}P^{o, *} \\
P^{n, *} \\
X_{p r e}^{o, *} \\
X^{o, *} \\
S^{o, *} \\
S^{n, *}=X^{n, *} \\
\pi^{*}\end{array}$ & $\begin{array}{l}- \\
\frac{c(1+\alpha)+1+\Delta}{2} \\
0 \\
0 \\
0 \\
\frac{1+\Delta-c(1+\alpha)}{2(1+\Delta)} \\
\frac{(c(1+\alpha)-(1+\Delta))^{2}}{4(1+\Delta)}\end{array}$ & $\begin{array}{l}- \\
\frac{(1+\Delta)(1+\beta-k)}{1+\beta} \\
0 \\
0 \\
0 \\
\frac{k}{1+\beta} \\
\frac{k((1+\beta)(\Delta+1-c(\alpha+1))-(\Delta+1) k)}{(1+\beta)^{2}}\end{array}$ & $\begin{array}{l}\frac{1}{2}(c+h+1) \\
\frac{1}{2}(c+h+1)+\Delta\left(1-\frac{k}{1+\beta}\right) \\
\frac{1-c-h}{2}-\frac{k}{1+\beta} \\
0 \\
\frac{1-c-h}{2}-\frac{k}{1+\beta} \\
\frac{k}{1+\beta} \\
\frac{(1+\beta)\left(\beta(c+h-1)^{2}+c^{2}+2 c(h-2 \alpha k-1)+4 h k+(h-1)^{2}\right)+4 \Delta k(\beta-k+1)}{4(1+\beta)^{2}}\end{array}$ \\
\hline$\pi^{*}$ & $(D): \bar{k}_{2} \leq k \wedge \tilde{\Delta}_{2}<\Delta<\tilde{\Delta}_{1}$ & $\begin{array}{l}(E):\left(\tilde{k}_{2} \leq k<\bar{k}_{2}\right) \wedge\left(\left(\hat{k}_{2}<k \wedge \beta>\right.\right. \\
\left.0) \vee\left(\tilde{\Delta}_{2}<\Delta \wedge \beta=0\right)\right) \wedge\left(\left(k>\hat{k}_{1} \wedge \Delta>\right.\right. \\
\left.\beta) \vee\left(k<\hat{k}_{1} \wedge \Delta<\beta\right) \vee\left(\Delta<\Delta_{1} \wedge \alpha=\beta\right)\right)\end{array}$ & $(F): \tilde{k}_{3} \leq k<\tilde{k}_{2} \wedge \tilde{\Delta}_{3}<\Delta<\tilde{\Delta}_{4}$ \\
\hline Rollover strategy & $\begin{array}{l}\text { dual sales rollover, dual } \\
\text { production rollover }\end{array}$ & $\begin{array}{l}\text { dual sales rollover, dual production } \\
\text { rollover }\end{array}$ & dual sales rollover, dual production rollover \\
\hline $\begin{array}{l}P^{o, *} \\
P^{n, *}\end{array}$ & $\begin{array}{l}\frac{c+1}{2} \\
\frac{c(1+\alpha)+1+\Delta}{2}\end{array}$ & $\begin{array}{l}\frac{\beta(-\alpha c+\beta(c+1))+\Delta(2(1-k)+\beta)}{2\left(\Delta+\beta^{2}\right)} \\
\frac{\Delta^{2}+(-\alpha c+\beta(c+1)) \beta+\Delta\left(-2(1+\beta) k+\left(c(\alpha-\beta)+2\left(1+\beta+\beta^{2}\right)\right)\right)}{2\left(\Delta+\beta^{2}\right)}\end{array}$ & $\begin{array}{l}\frac{1+c+h}{2} \\
\frac{1+\Delta+c(1+\alpha)+h(1+\beta)}{2}\end{array}$ \\
\hline $\begin{array}{l}X_{p r e}^{o, *} \\
X^{o, *} \\
S^{o, *} \\
S^{n, *}=X^{n, *} \\
\pi^{*}\end{array}$ & $\begin{array}{l}0 \\
\frac{c(\alpha-\Delta)}{2 \Delta} \\
\frac{c(\alpha-\Delta)}{2 \Delta} \\
\frac{\Delta-\alpha c}{2 \Delta} \\
\frac{c^{2} \Delta+\alpha^{2} c^{2}-2 \alpha c \Delta-2 c \Delta+\Delta^{2}+\Delta}{4 \Delta}\end{array}$ & $\begin{array}{l}0 \\
\frac{(1+\beta)(c(\alpha-\beta)+(\beta-\Delta))+2 k(\Delta-\beta)}{2\left(\Delta+\beta^{2}\right)} \\
\frac{(1+\beta)(c(\alpha-\beta)+(\beta-\Delta))+2 k(\Delta-\beta)}{2\left(\Delta+\beta^{2}\right)} \\
\frac{2 \beta k+(c(\beta-\alpha)+\Delta-\beta)}{2\left(\Delta+\beta^{2}\right)} \\
\frac{\beta^{2}(c-1)^{2}+\Delta^{2}-4 \Delta k(c-1+k)-2 c \Delta \alpha+c^{2} \alpha^{2}+2 \beta(c-1+2 k)(\Delta-c \alpha)}{4\left(\Delta+\beta^{2}\right)}\end{array}$ & $\begin{array}{l}\frac{\Delta(1+\beta)-c(\Delta+\alpha \beta)-h\left(\Delta+\beta^{2}\right)}{2 \Delta}-k \\
\frac{(1+\beta)(c \alpha+h \beta)+\Delta(2 k-(\beta+1))}{2 \Delta} \\
\frac{c(\alpha-\Delta)+h(\beta-\Delta)}{2 \Delta} \\
\frac{\Delta-\alpha c-h \beta}{2 \Delta} \\
\frac{\Delta^{2}+\Delta\left(1+c^{2}+h(-2-2 \beta+h+4 k)+2 c(-1+h-\alpha)\right)+(\beta h+c \alpha)^{2}}{4 \Delta}\end{array}$ \\
\hline$\pi^{*}$ & $(G): \bar{k}_{3} \leq k \wedge \Delta \leq \tilde{\Delta}_{2}$ & $\begin{array}{l}(H):\left(\tilde{k}_{4} \leq k<\bar{k}_{3}\right) \wedge\left(\left(k \leq \hat{k}_{2} \wedge \beta>\right.\right. \\
0) \vee\left(\Delta \leq \tilde{\Delta}_{2} \wedge \beta=0\right)\end{array}$ & $(I): k<\tilde{k}_{4} \wedge \Delta \leq \tilde{\Delta}_{3}$ \\
\hline Rollover strategy & do not introduce & do not introduce & do not introduce \\
\hline $\begin{array}{l}P^{o, *} \\
P^{n, *} \\
X_{p r e}^{o, *} \\
X^{o, *} \\
S^{o, *} \\
S^{n, *}=X^{n, *} \\
\pi^{*}\end{array}$ & $\begin{array}{l}\frac{c+1}{2} \\
- \\
0 \\
\frac{1-c}{2} \\
\frac{1-c}{2} \\
0 \\
\frac{(c-1)^{2}}{4}\end{array}$ & $\begin{array}{l}1-k \\
- \\
0 \\
k \\
k \\
0 \\
(1-c-k) k\end{array}$ & $\begin{array}{l}\frac{c+h+1}{2} \\
- \\
\frac{1-c-h}{2}-k \\
k \\
\frac{1-c-h}{2} \\
\frac{(c+h-1)(1+3 h-c)}{4}+h k\end{array}$ \\
\hline
\end{tabular}

Table 3

Firm's optimal decisions and resulting profit if capacity to pre-produce is binding.

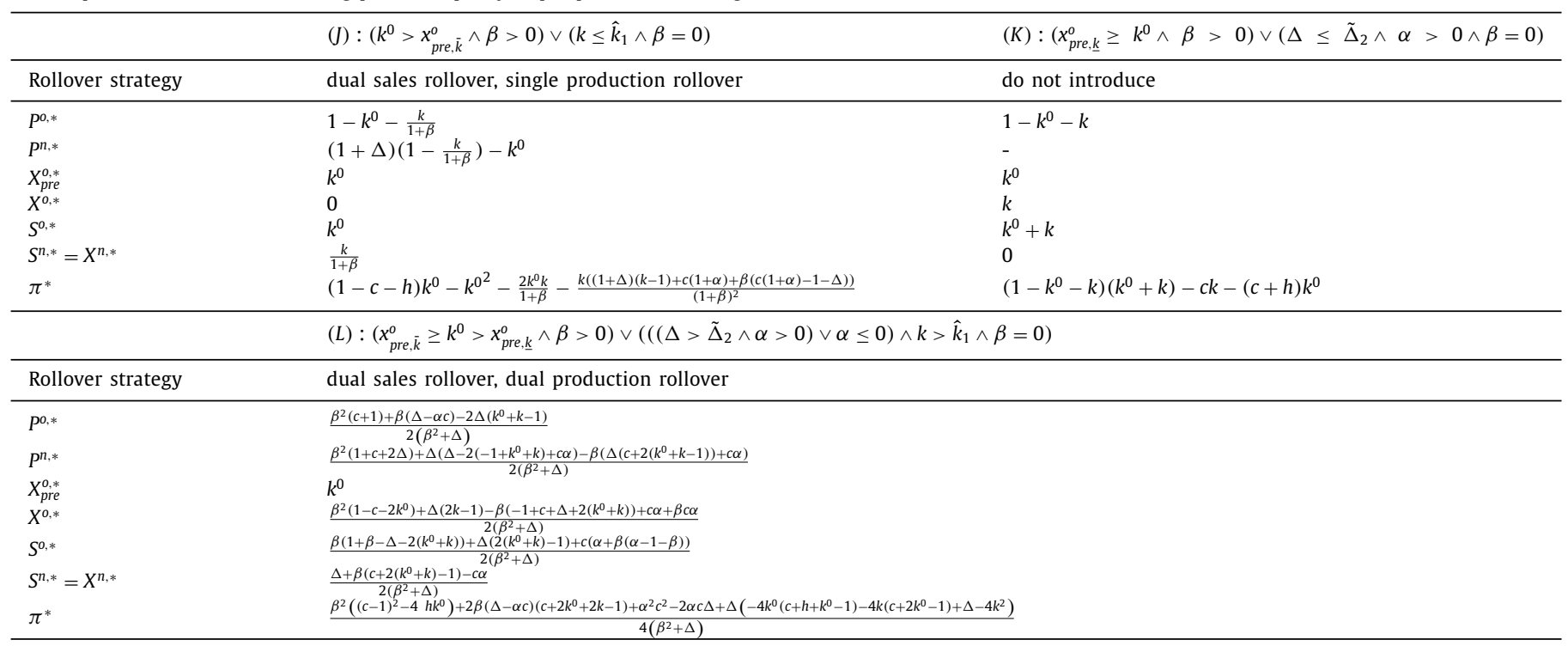




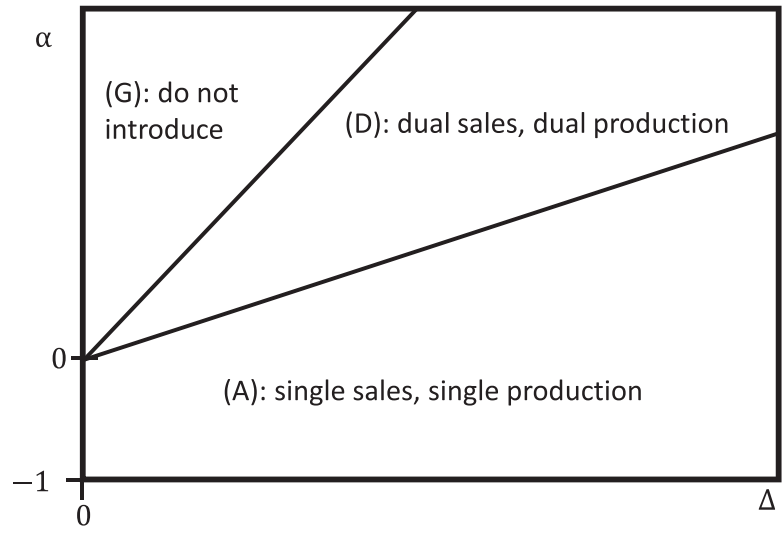

Fig. 3. Optimal rollover strategies for non-binding capacity $k>\bar{k}$ during the introduction period depending on the difference in variable production costs $\alpha$ and the difference in quality $\Delta$.

\section{Results and discussion}

Based on the analysis in the preceding section, we discuss the optimal decisions and selected rollover strategies in two subsections depending on the available production capacity during the introduction period as well as the impact of limited pre-production capacity. This section is concluded by a numerical investigation of the impact of the mitigation actions on the obtainable profit.

\subsection{Optimal sales and production rollover strategies for sufficient capacity}

We first establish the condition under which there is sufficient capacity such that the optimal decisions are independent of the production capacity $k$. Subsequently, we analyze the resulting optimal rollover strategies.

Corollary 1. The capacity $\bar{k}$ that is required such that the optimal decisions are independent of any capacity $k \geq \bar{k}$ is

$\bar{k}= \begin{cases}\bar{k}_{1} & \tilde{\Delta}_{1}<\Delta, \\ \bar{k}_{2} & \tilde{\Delta}_{2}<\Delta \leq \tilde{\Delta}_{1}, \\ \bar{k}_{3} & \Delta \leq \tilde{\Delta}_{2}\end{cases}$

The value of $\bar{k}$ depends on the relative difference in the quality $\Delta$ where $\tilde{\Delta}_{1}$ marks the threshold between SSR and DSR and $\tilde{\Delta}_{2}$ marks the threshold between DSR and the case where the new product is not introduced as given by Theorem 1 . For capacity $k>\bar{k}$, the optimal rollover strategy choice is depicted in Fig. 3. It depends on the relative difference in quality $\Delta$ and the relative difference in production costs $\alpha$.

Observation 1. If there is sufficient capacity $(k \geq \bar{k})$, the production and sales rollover strategies are always aligned.

Fig. 3 illustrates Observation 1. Moreover, it shows the dominance of the SSR strategy if the product generations have the same marginal production costs, or the new generation is cheaper to manufacture $(\alpha \leq 0)$. Intuitively, the new product is more attractive for customers due to increased quality $(\Delta>0)$ and allows for an at least equal profit margin for the firm. This confirms the findings by Levinthal and Purohit (1989) and Li and Graves (2012) with respect to the impact of marginal production costs on the selection of sales rollover strategy for unlimited production capacity.

In the case of a marginal production costs increase $(\alpha>0)$, the minimum increase in quality such that the firm will introduce the new generation is $\tilde{\Delta}_{2}=c \alpha$. The firm then chooses a DSR and a DPR strategy. For new generations with $\Delta>\alpha=\tilde{\Delta}_{1}$, an SSR and
SPR become optimal. These findings are in line with the finding of Koca et al. (2010) that a larger difference between product generations drives the firm towards an SSR strategy.

\subsection{Optimal mitigation actions for binding capacity during the introduction period}

In the case that the available capacity of the firm is below $\bar{k}$, i.e., the capacity constraint (2e) is binding, it cannot produce the required amount of products to fulfill the transition as discussed in Section 5.1. We analyze the optimal actions to mitigate the effects of capacity shortages and the actions' dependence on the severity of the capacity shortage, as well as on the relative differences in quality, variable production costs, and capacity consumption.

Since we are interested in capacity shortages that occur during the introduction of a new product generation, the following discussion focuses on cases where available capacity is sufficient if only the old product is produced and sold:

$k \geq \bar{k}_{3}=\frac{1-c}{2}$.

Moreover, the analysis focuses on cases where the increase in quality is large enough to justify the introduction of the new generation:

$\Delta>\tilde{\Delta}_{2}=c \alpha$.

In addition, Theorem 1 shows that if it is not optimal to introduce a new generation, given sufficient capacity $k \geq \bar{k}_{3}$, that is, $\Delta \leq \tilde{\Delta}_{2}$, it is never optimal to introduce the new generation for binding capacity $k<\bar{k}_{3}$ and $\Delta \leq \tilde{\Delta}_{2}$. Conditions (4) and (5) exclude the regions $(G),(H),(I)$, and $(K)$ in which the new generation is not introduced at all.

In order to account for limited capacity, the firm can change its production, sales, and pricing decisions. This may also require a change in the sales and the production rollover strategy. The optimal values of the decisions on production, sales, and pricing depend on the level of available capacity and the product parameters.

The analytical results indicate that the firm can take two types of mitigation actions to reduce the negative effect of capacity shortages, depending on the severity of the capacity shortage and the product parameters:

(i) Changing prices while maintaining the sales and production rollover strategy that is optimal for the non-binding capacity case $(k \geq \bar{k})$.

(ii) Changing the sales and/or production rollover strategies and exploiting pre-production supported by changes in prices of the old and the new generation.

The applicability of the two mitigation actions given above is described in the following two subsections.

\subsubsection{Mitigating minor capacity shortages}

If the capacity during the introduction period is binding, the firm has to react with changes in the pricing of the product.

Corollary 2. The prices $P^{o}$ and $P^{n}$ are decreasing in the available capacity $k$.

The increase in prices of the sold product generations for decreasing capacity reflects the market power of the monopolist. However, this does not imply that the price for the old generation increases in the introduction period compared to the price before the introduction period. The quality level of a product $q$ is typically a decreasing function of time. Hence, the pricing before the introduction period is typically higher to exploit the higher quality value. 


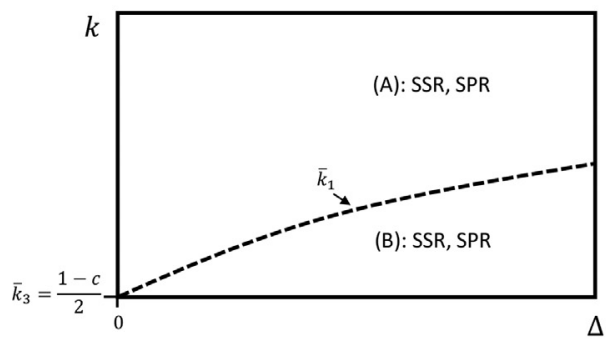

(a) $\alpha \leq 0$

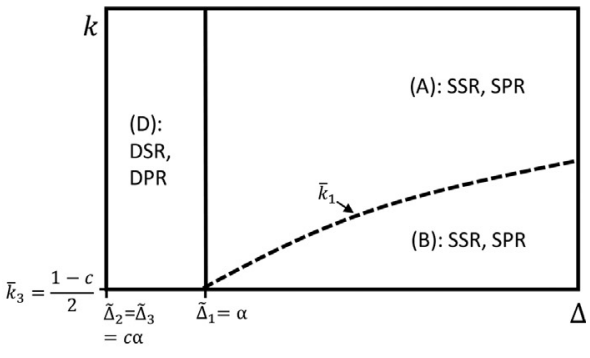

(b) $\alpha>0$

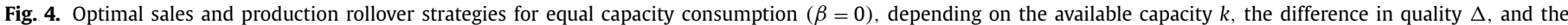
difference in variable production costs $\alpha$, with regions $(A),(B)$, and $(D)$ as given in Table 2 .

Observation 2. For minor capacity shortages, it is sufficient to only use pricing as mitigation action.

If $k$ is close to $\bar{k}$ it is sufficient to use pricing such that the resulting demand is equivalent to the available capacity. This applies to all cases for which $k$ is limited from above by $k$. Furthermore, if the old and the new generation consume the same amount of capacity per produced unit, the following result can be obtained.

Corollary 3. In the region of interest $\bar{k}_{3} \leq k<\bar{k} \wedge \Delta>\tilde{\Delta}_{2}$, if there is no difference in the capacity consumption $\beta=0$, the optimal sales rollover strategy and the optimal production rollover strategies are always equivalent to the optimal strategies for sufficient capacity $k>\bar{k}$.

Fig. 4 illustrates the case of $\beta=0$ by depicting optimal sales and production rollover strategies given by Theorem 1 for $k \geq \bar{k}_{3}$ and $\Delta>\tilde{\Delta}_{2}$. It shows that pricing is sufficient in the complete parameter range of interest. As available capacity decreases, mitigation via pricing always corresponds to a change from the region $(A)$ to the region $(B)$ while maintaining a SSR and a SPR. A change in price is sufficient for $\bar{k}_{3} \leq k<\bar{k}$ because there is a quality advantage for the new generation but no quantity advantage for the old product because the old and the new product generation consume the same amount of capacity. Therefore, switching from an SPR to a DPR strategy is not beneficial. Moreover, the additional revenue from old products, in the case of a DSR and an SPR with pre-produced old products, cannot recover the inventory holding costs and the losses from cannibalization.

\subsubsection{Mitigating severe capacity shortages}

In the following, we describe the mitigation action for severe capacity shortages during the introduction period such that reacting by changes in pricing is insufficient to mitigate the shortages. The second type of mitigation actions encompasses the change of the sales and/or production rollover strategies and exploiting preproduction supported by changes in prices of the old and the new generation. In this subsection, we analyze settings in which the constraint on the firm's capacity $k^{0}$ to pre-produce the old generation is not binding. This is often the case, as sales and production decrease at the end of a product lifecycle. Implications of insufficient capacity to pre-produce are discussed in the succeeding subsection. In general, we make the following observation.

Observation 3. Decreasing capacity drives the firm towards a DSR for two reasons:

(i) A DSR allows for a DPR strategy, which increases the quantity of products that can be produced during the introduction period because the old product is less capacity intensive.

(ii) A DSR facilitates exploiting pre-production before the introduction period.

Whether changes in the rollover strategy or pre-production are the optimal mitigation actions depends on the relation between the relative capacity increase and the relative difference in variable production costs, i.e., $\alpha \leq \beta$ or $\alpha>\beta$, and the relative increase in quality $\Delta$.

Fig. 5 visualizes the optimal sales and production rollover strategies given by Theorem 1 for $\beta>0, \alpha \geq 0, \alpha \leq \beta$, in the region of interest $k>\bar{k}_{3}, \Delta>\tilde{\Delta}_{2}$, and $h<\frac{1-c}{1+\frac{1}{\beta}}$, such that the necessary condition for the use of pre-production is fulfilled. The shaded areas mark combinations of $k$ and $\Delta$ for which a change in sales and/or production rollover strategies compared to the case $k \geq \bar{k}$ is optimal. Cases with $\alpha<0$ are not depicted because they resemble the case depicted in Fig. 5a. The only structural difference is that the threshold $\tilde{\Delta}_{4}$ moves to smaller values of $\Delta$ and disappears for $\alpha<-\frac{h \beta}{c}$. The case of $\beta=0$ is covered by Corollary 3 in Section 5.2.1.

It can be observed that the optimal mitigation actions differ structurally depending on the relative quality increase $\Delta$. We differentiate the three cases of a high $\left(\Delta \geq \tilde{\Delta}_{4}\right)$, a medium $\left(\tilde{\Delta}_{1}<\right.$ $\left.\Delta<\tilde{\Delta}_{4}\right)$, and a low $\left(\tilde{\Delta}_{2} \leq \Delta \leq \tilde{\Delta}_{1}\right)$ quality increase.

New products with high quality increase.

For new generations within the high-quality range $\left(\Delta \geq \tilde{\Delta}_{4}\right)$, an SPR strategy is always optimal independent of the capacity $k$. The quality of the new generation and the prices that can be charged are so large that it is always optimal to devote the complete capacity during the introduction period to the new generation. However, for severe capacity shortages with a capacity $k$ below the threshold $\tilde{k}_{1}$, it is optimal to switch from an SSR strategy in region $(B)$ to a DSR strategy in region $(C)$.

Observation 4. For severe capacity shortages, it can be optimal to misalign production rollover strategy (SPR) and sales rollover strategy (DSR).

This misalignment is realized via pre-production that is beneficial as long as inventory holding costs are below $h<\frac{1-c}{1+\frac{1}{\beta}}$. The increased revenues due to sales of the pre-produced old products overcompensate for the inventory holding costs and the cannibalization of the new generation.

New products with medium quality increase.

The medium quality increase in the range of $\tilde{\Delta}_{1}<\Delta<\tilde{\Delta}_{4}$ exists only for $\alpha<\beta$. For $\alpha=\beta$, the thresholds $\tilde{\Delta}_{1}$ and $\tilde{\Delta}_{4}$ coincide (Fig. 5c). If mitigation via prices is insufficient because the capacity falls below $k<\hat{k}_{1}$, a change from an SSR and an SPR $(B)$ to a DSR and a DPR strategy $(E)$ is optimal. In this situation, the cannibalization of sales of the new generation by the old is not a major concern for the firm because the available capacity limits the quantity of the new generation that can be produced and sold. Instead, switching to a DSR and a DPR strategy allows for increased revenues from the old generation, which requires less capacity per 


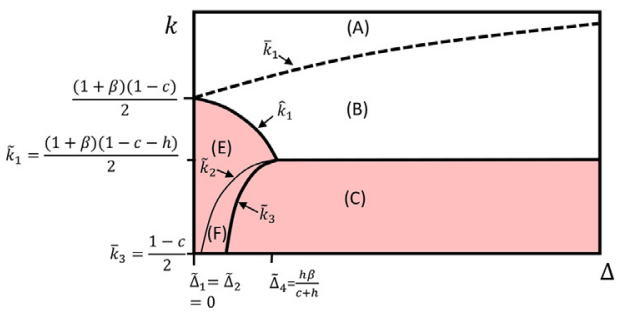

(a) $\alpha=0,0<\beta$

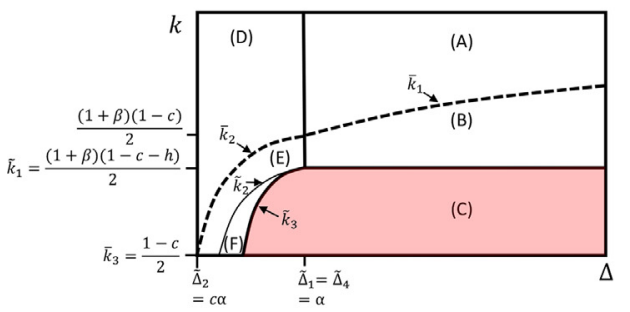

(c) $0<\alpha=\beta$

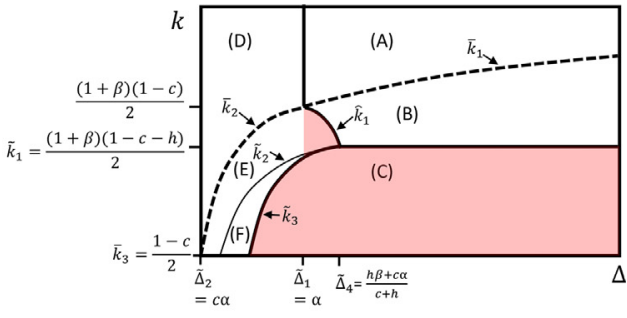

(b) $0<\alpha<\beta$

changed sales and/ or production rollover strategy compared to $\mathrm{k}>\bar{k}$

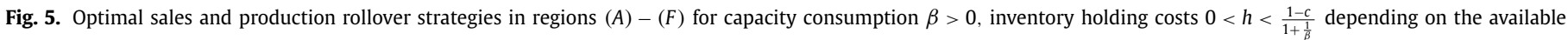
capacity $k$, the difference in quality $\Delta$, and the difference in variable production costs $\alpha$.

product. Therefore, more products can be produced with the available capacity. In this case, the quantity increase outweighs the negative effects of cannibalization and lower prices for the old generation. If capacity shortages are more severe $\left(k<\tilde{k}_{2}\right)$ and the holding costs are not prohibitively high $\left(h<\frac{1-c}{1+\frac{1}{\beta}}\right)$, the firm starts to use pre-production while maintaining a DSR and a DPR strategy $(F)$. In this case, the production in the introduction period shifts from the old to the new generation, such that the sales of the new generation $S^{n, *}$ are not affected by decreasing capacity. The reduced production of the old generation is compensated for by the preproduction, such that the same amount of sales of the old generation is maintained during the introduction period. Ultimately the complete amount of sold old products is pre-produced if $k$ falls below $\tilde{k}_{3}$ (region $(C)$ ). This leads to the following observation.

Observation 5. For products with medium quality increase, the optimal production rollover strategy is non-monotone in the capacity, i.e., for decreasing capacity the optimal mitigation actions are changes from a SPR to a DPR and back to a SPR.

New products with low quality increase.

The range of new generations with low quality $\left(\tilde{\Delta}_{2} \leq \Delta \leq \tilde{\Delta}_{1}\right)$ exists only for $\alpha>0$; otherwise $\tilde{\Delta}_{2}$ coincides with the threshold $\tilde{\Delta}_{1}$ (Fig. 5a). The main difference to the medium quality case is that for sufficient capacity $k \geq \bar{k}$ a DSR and a DPR strategy are optimal (region $(D)$ ). Hence, a shift to region (E) in response to capacity shortages means only mitigation via pricing but no change in rollover strategies. Otherwise, the mitigation actions are the same as for products with a medium quality increase.

New products with larger relative increase in variable production costs that exceeds the relative increase in capacity consumption.

We now turn to cases where the relative increase in variable production costs exceeds the relative increase in capacity consumption $(\alpha>\beta)$. The resulting optimal sales and production rollover strategies are depicted in Fig. 6 for the case that $h<\frac{1-c}{1+\frac{1}{\beta}}$.

Since $\tilde{\Delta}_{4}<\tilde{\Delta}_{1}$ in the case of $\alpha>\beta$, we now refer to products in the ranges of $\Delta \leq \tilde{\Delta}_{4}$ as low quality, $\tilde{\Delta}_{4}<\Delta<\tilde{\Delta}_{1}$ as medium quality, and $\Delta \geq \tilde{\Delta}_{1}$ as high quality. The optimal mitigation actions for new generations in the low-quality range and high-quality range follow the same structure as discussed for $\alpha \leq \beta$. How-

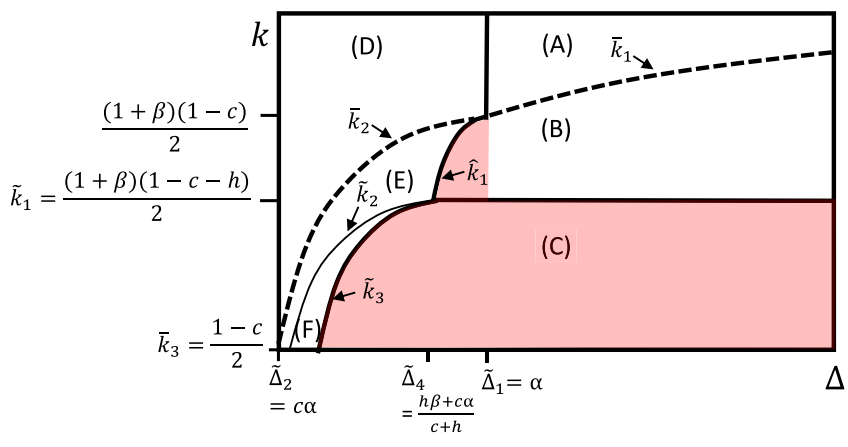

changed sales and/ or production rollover strategy compared to $\mathrm{k}>\bar{k}$

-ேーーー $\bar{k}$

Fig. 6. Optimal sales and production rollover strategies in regions $(A)-(F)$ for inventory holding costs $0<h<\frac{1-c}{1+\frac{1}{\beta}}$ and an increase in variable production costs greater than the increase in capacity consumption $\alpha>\beta$ depending on the available capacity $k$ and the difference in quality $\Delta$.

ever, the optimal mitigation strategies for new generations in the medium-quality range $\tilde{\Delta}_{4}<\Delta<\tilde{\Delta}_{1}$ are different.

It might be assumed that for cases in which variable production costs and capacity consumption increase for the new product $(\alpha>0, \beta>0)$, a switch due to limited capacity from a DSR and a DPR strategy to an SSR and an SPR strategy without pre-production is never optimal because this switch means shifting the production exclusively towards the generation that is more expensive to produce, and at the same time more demanding in terms of the capacity consumption. However, we find that a shift from a DPR and a DSR strategy in the region (E) to an SPR and an SSR in region $(B)$ is optimal if the capacity falls below the threshold $\hat{k}_{1}$ for $0<\alpha<\beta$ and there is medium quality increase $\left(\tilde{\Delta}_{4}<\Delta<\tilde{\Delta}_{1}\right)$ (see Fig. 6). This at first sight counterintuitive finding is driven by the cannibalization between product generations. By removing the old product, the firm is able to charge higher prices since pricesensitive customers cannot substitute with the old generation. This is particularly relevant in this setting because of the high value of 
$\alpha>\beta$ that in turn requires a high price $P^{n}$ to make the sales of the new generation profitable.

A change back from an SSR $(B)$ to a DSR strategy $(C)$ occurs if the capacity falls below $\tilde{k}_{1}$ and $h<\frac{1-c}{1+\frac{1}{\beta}}$. In this case, the capacity is so tight, that the benefits of selling pre-produced old products overcompensate for the associated holding costs and cannibalization. This leads to the following observation.

Observation 6. For products with medium quality increase, the optimal sales rollover strategy is non-monotone in the capacity, i.e., for decreasing capacity the optimal mitigation actions are changes from an DSR to a SSR and back to an DSR.

In conclusion, we find that severe shortages in the production capacity during the introduction period require changes in the sales and production rollover strategies. In contrast to the existing literature, we find that in the case of limited capacity, it can be optimal to deliberately choose unaligned sales rollover and production rollover strategies. Even for new generations with a substantial increase in quality, a DSR in combination with an SPR can be optimal because it allows for the exploitation of pre-production if the available capacity is scarce.

\subsubsection{Impact of binding pre-production capacity}

A finite pre-production capacity can affect only parameter regions in which under unlimited pre-production capacity it is optimal to pre-produce, i.e., regions $(\mathrm{C}),(\mathrm{F}),(\mathrm{I})$. Hence, the finding of Corollary 3 is not affected by limited pre-production capacity. That is, for equal capacity consumption of both generations $(\beta=0)$ pricing is always a sufficient mitigation action. Theorem 1 shows that if pre-production capacity becomes binding (Constraint (2d)), the optimal approach of the firm is to use all of the available capacity $k^{0}$.

Concerning pricing, a smaller capacity during the introduction period $k$ also leads to higher prices if the ability to pre-produce is limited, i.e., Corollary 2 includes the regions $(J),(K)$, and $(L)$. Moreover, the prices also increase if $k^{0}$ is decreased. Intuitively, the price of the old product is increased to match the available units of the old product with demand. The increased price of the old generation reduces the cannibalization of the new generation. To take advantage of this, it is optimal to also increase the price of the new generation.

These changes in optimal decisions are also reflected in the optimal rollover strategies. The pre-production capacity limits the firm's ability to misalign sales and production rollover strategy. Therefore, the second reason for the usage of DSR strategy as stated in Observation 3 is affected by limited pre-production capacity. In the extreme case of $k^{0}=0$, it is no longer possible to combine a DSR with an SPR, i.e., Observations 4 and 5 cannot be made. However, the finite production capacity $k$ may still require a change in the sales and production rollover strategy compared to the uncapacitated setting discussed in Section 5.1.

Fig. 7 depicts the optimal sales and production decisions given unlimited pre-production capacity $\left(k^{0}=\infty\right)$ and the extreme case that no pre-production is possible $\left(k^{0}=0\right)$ depending on the production capacity during the introduction period. The optimal decisions only deviate for $k<\tilde{k}_{2}=0.32$ for which pre-production is optimal, given $k^{0}=\infty$. For the case $k^{0}=0$ pre-production cannot occur, hence the firm cannot combine a DSR with an SPR for $k<\tilde{k}_{3}=0.28$. Instead, it is optimal to stick to a DSR and a DPR strategy to mitigate the effects of the limited capacity. Note that this still implies a change in the optimal rollover strategy compared to the uncapacitated cases for which an SSR and an SPR are optimal.

\subsection{Impact of limited capacity and mitigation actions on the profit}

Obviously, the smaller the available capacity $k$ the lower the profit that can be obtained because Constraint (2e) becomes more restrictive. This section provides insights into how important the optimal mitigation actions, as described in the previous section, are with respect to the obtainable profit. Therefore, we compare the optimal solutions to a common but simplified sequential planning approach.

The sequential planning approach first determines the sales rollover strategy and pricing decisions under the assumption that there will be sufficient capacity. In the second step, the firm tries to fulfill the sales plan under the actual available capacity. For example, Erhun et al. (2007) report that Intel made pricing decisions without considering available production capacities that led to substantial problems during the introduction period.

In the first step of the sequential planning approach, it is assumed that unlimited capacity before and during the introduction period is available $\left(k^{0}=k=\infty\right)$. The optimal amounts of old and new products to be sold $S_{\infty}^{0, *}, S_{\infty}^{n, *}$ and the prices of the old and the new generation $P_{\infty}^{0, *}, P_{\infty}^{n, *}$ are obtained according to Lemma 3 given in Appendix B. The prices for the old and the new generation are set to the solution obtained for the non-binding capacity case:

$P^{o, s e q}=P_{\infty}^{o, *}$ and $P^{n, s e q}=P_{\infty}^{n, *}$.

We consider two alternatives to account for the finite capacity $k$ in the second step, first a proportional cut of the production quantities and second a reoptimization of the production quantity subject to the available capacity.

The proportional reduction of the production quantities ensures that the original sales rollover strategy is preserved. The production quantities are set in such a way that they match the capacity constraint (2e):

$S^{o, \text { seqPro }}=X^{o, \text { seqPro }}=k \frac{S_{\infty}^{o, *}}{S_{\infty}^{0, *}+(1+\beta) S_{\infty}^{n, *}}$,
$S^{n, \text { seqPro }}=X^{n, \text { seqPro }}=k \frac{S_{\infty}^{n, *}}{S_{\infty}^{0, *}+(1+\beta) S_{\infty}^{n, *}}$.

The second variant is a reoptimization of the production quantities by the production department in the second step. For given prices according to (6), the optimization problem (2) can be transformed into a mixed-integer program that is used to obtain the optimal production and sales quantities $S^{o, s e q 0 p t}=X^{o, s e q 0 p t}$ and $S^{n, s e q 0 p t}=$ $X^{n \text {,seqopt }}$. However, this reoptimization may lead to a change in the sales and production rollover strategies that were initially intended in the first step.

To evaluate the losses if the limited capacity is ignored in the first planning step, we compare the optimal solution given in Theorem 1 to the two variants of the sequential planning approach. The relative profit losses $\delta^{\text {seqPro }}=\frac{\pi^{*}-\pi^{\text {seqPro }}}{\pi^{*}}$ and $\delta^{\text {seqOpt }}=\frac{\pi^{*}-\pi^{\text {seqOpt }}}{\pi^{*}}$ are calculated, given the optimal profit $\pi^{*}$ and the profits $\pi^{\text {seqPro }}$, $\pi^{\text {seqOpt }}$, respectively.

Fig. 8 shows the relative profit losses for both sequential approaches depending on the available capacity $k$ and the relative increase in quality $\Delta$ that satisfy Conditions (4) and (5) for the exemplary numerical values $\alpha=0.2, \beta=0.5, c=0.5, h=0.05, k^{0}=$ $\infty$.

For both approaches, the relative profit loss $\delta$ increases with an increasing difference in the quality $\Delta$ between generations and with decreasing capacity $k$. The greatest profit losses are observed for cases with $\Delta>\tilde{\Delta}_{4}=0.23$ and $k<\tilde{k}_{1}=0.34$ in which the optimal sales rollover strategy is changed from an SSR to a DSR. The optimization of the production quantities in the second stage improves only the profit for new generations with lower quality $\Delta<\tilde{\Delta}_{1}$ for which under unlimited capacity a DSR is optimal (Fig. 8b). 


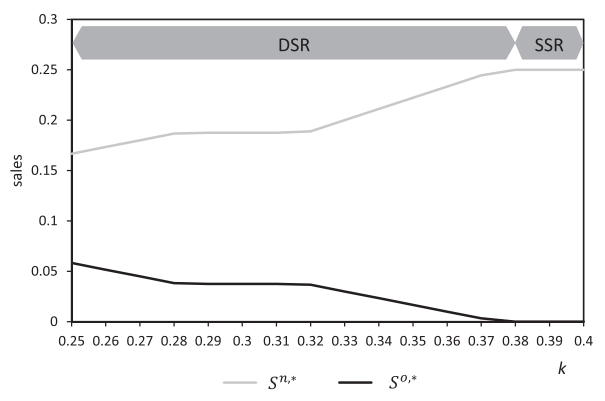

(a) Optimal sales decisions for $k^{0}=\infty$

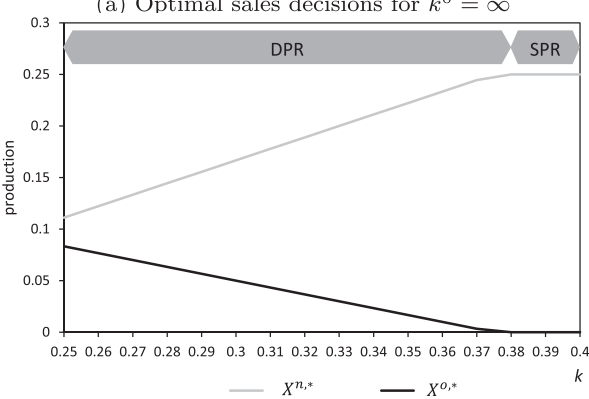

(c) Optimal sales decisions for $k^{0}=0$

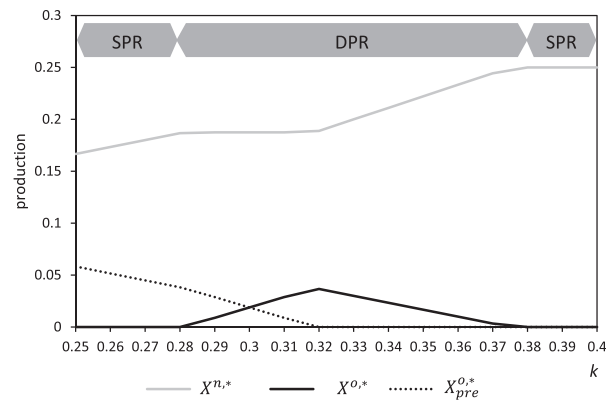

(b) Optimal production decisions for $k^{0}=\infty$

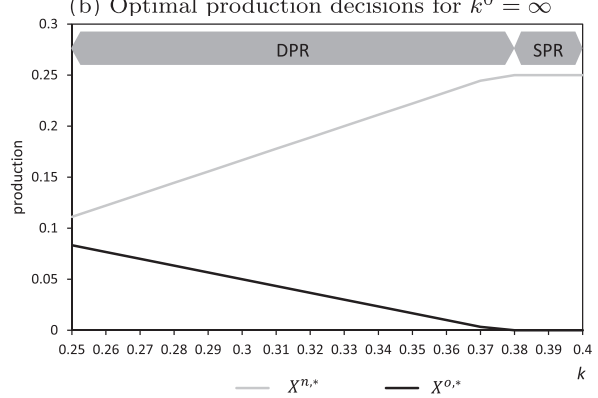

(d) Optimal production decisions for $k^{0}=0$

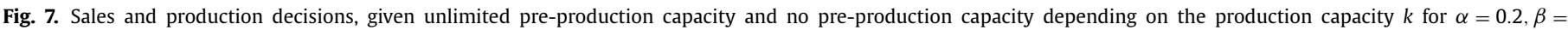
$0.5, \Delta=0.2, c=0.5, h=0.05$.

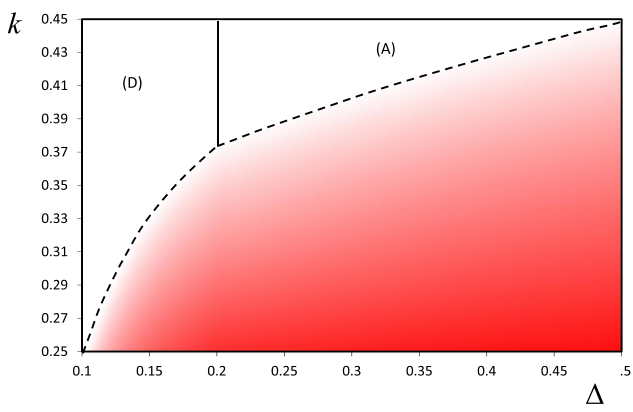

(a) Proportional production

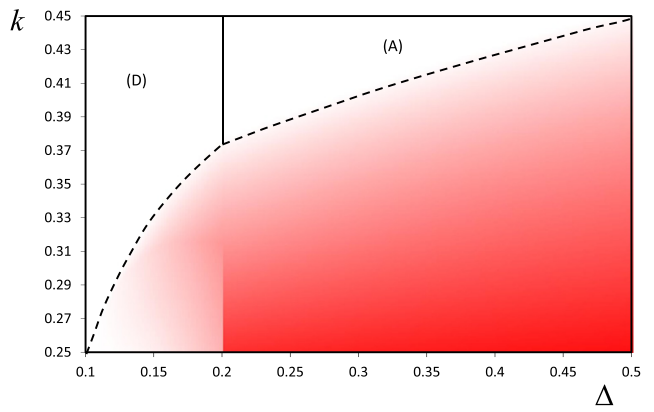

(b) Reoptimized production

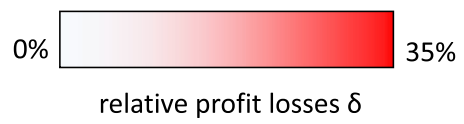

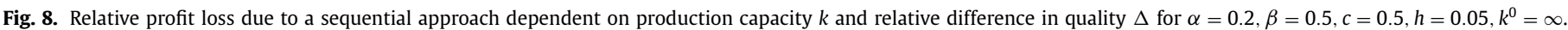

An additional numerical study in which the firm does not have the option of pre-production, i.e., $k^{0}=0$, yielded structurally equivalent results.

The presented numerical comparisons show that substantial profit losses can occur if firms use a sequential planning approach compared to the optimal integrated approach in which all decisions are made under the explicit consideration of the limited capacity.

\section{Managerial insights}

Based on the discussion in the previous section, we highlight the practical implications for the management of product rollovers under capacity constraints. Key drivers for the selection of the rollover strategy are the available capacity and the difference in quality between the product generations. Table 4 qualitatively summarizes the optimal rollover strategy selection based on these two parameters and the increase in capacity consumption of the new generation.

- If the old generation and the new generation consume the same amount of capacity per produced unit during the introduction period, the selection of the sales and production rollover strategy mainly depends on the increase in quality. For products with a large quality increase, an SSR in combination with an SPR is optimal. The use of a DSR aligned with a DPR is optimal for new generations with a lower increase in quality. Limited capacity is always mitigated via pricing without the need for a change in the rollover strategies.

- In contrast, if the new generation consumes more capacity than the old generation, changes in the sales and production strategy are beneficial in case of severe capacity shortages.

For introductions of new generations with a small quality increase, severe capacity shortages drive the firm to combine a DSR with an SPR. The SPR frees up capacity for the new gen- 
Table 4

Optimal rollover strategies depending on capacity and quality increase.

(a) Equal capacity consumption of both generations

\begin{tabular}{|c|c|c|c|}
\hline & & \multicolumn{2}{|l|}{ Quality increase } \\
\hline & & low & high \\
\hline \multirow[t]{2}{*}{ Capacity during introduction } & high & $\begin{array}{l}\text { dual sales } \\
\text { dual production }\end{array}$ & $\begin{array}{l}\text { single sales } \\
\text { single production }\end{array}$ \\
\hline & low & $\begin{array}{l}\text { dual sales } \\
\text { dual production }\end{array}$ & $\begin{array}{l}\text { single sales } \\
\text { single production }\end{array}$ \\
\hline
\end{tabular}

(b) Increased capacity consumption of the new generation

\begin{tabular}{|c|c|c|c|}
\hline & & \multicolumn{2}{|l|}{ Quality increase } \\
\hline & & low & high \\
\hline \multirow[t]{2}{*}{ Capacity during introduction } & high & $\begin{array}{l}\text { dual sales } \\
\text { dual production }\end{array}$ & $\begin{array}{l}\text { single sales } \\
\text { single production }\end{array}$ \\
\hline & low & $\begin{array}{l}\text { dual sales } \\
\text { single production }\end{array}$ & $\begin{array}{l}\text { dual sales } \\
\text { single production }\end{array}$ \\
\hline
\end{tabular}

eration while the demand for the old product is still fulfilled via pre-produced products. For new generations with a high quality increase, the switch from an SSR to a DSR supported by pre-production is also optimal. The capacity is more restrictive to the sales of the new generation than the reduction due to cannibalization effects induced by the old generation. Hence, independently of the quality increase, it is optimal for severe capacity shortages to mix an SPR with a DSR. The limited capacity is focused on the new generation while it is possible to sell products to a broad range of the market by the exploitation of pre-production.

- Whereas the optimal mitigation actions for capacity shortages are relatively straightforward for low and high quality increases, the optimal mitigation actions are more complex for medium quality increases of the new product. Surprisingly, the optimal strategy selection for sales and production rollover is nonmonotone in the available capacity. This means a switch in the strategy that is optimal to react to medium capacity shortages may need to be reversed for more severe capacity shortages. This is due to the complex interdependencies between the customer behavior and the operational parameters of the firm.

For example, if the relative increase in capacity consumption exceeds the relative increase in variable production costs, it is beneficial to switch from an SPR and an SSR to a DPR and a DSR in response to medium capacity shortages. This change makes use of the capacity efficiently because per capacity unit more products are produced. However, if the capacity shortages are more severe it is beneficial to switch back to an SPR and devote the complete capacity to the new product while fulfilling the demand for the old generation by pre-production.

- If the relative increase in variable production costs exceeds the relative increase in capacity consumption it is optimal to switch from a DSR and a DPR to an SSR and an SPR. This strategy switch may seem counterintuitive at first sight because the complete sales and production are shifted to the product that has the higher variable costs and consumes more capacity per unit. However, it allows the firm to charge a higher price for the limited amount of products that can be produced with the available capacity. This premium price could not be charged in the presence of the old product on the market that causes cannibalization effects. Only if the capacity shortages are so severe such that they restrict the sales of the new generation instead of the substitution effects, it is optimal to utilize a DSR in combination with an SPR supported by pre-production.
- Choosing the optimal mitigation actions is important because rollover strategies and pricing decisions that are derived under the assumption of unlimited capacity may lead to substantial profit losses if applied to cases in which the capacity is scarce. These losses increase with the severity of the capacity shortage and the increase of the quality of the new generation.

\section{Conclusions}

In this paper, we analyzed a capacity-constrained monopolist that intends to introduce a new product in a market in which a predecessor generation is already sold. The firm selects sales and production rollover strategies and decides the amount of the old and the new products to be produced and to be sold as well as the corresponding prices to maximize profit.

The proposed model generalizes existing operations models by incorporating the selection of sales and production rollover strategies and price decisions. Although there are models for the selection of rollover strategies, the existing models ignore capacity constraints that are often observed during new product introductions. Our model includes both the selection of rollover strategies and finite capacity to capture the interdependencies between the sales and operations-related decisions.

Our first contribution is to derive the firm's optimal decisions and the structure of the optimal combination of sales and production rollover strategies.

We identify two different mitigation actions in response to limited capacity. First, increased prices can be used to match demand to the available capacity, and at the same time exploiting the customers' willingness to pay, in order to sustain profits. Second, additional changes in the sales and/or production rollover strategy, as well as pre-producing the old generation, can be required.

We establish that limited capacity requires a differentiation between sales and production rollover strategies because unlike in the case of unlimited capacity, sales and production rollover strategies are not necessarily aligned. We show that combining a DSR with an SPR strategy can be optimal. Moreover, we find that limited capacity drives the firm to select a DSR strategy if the increase in capacity consumption exceeds the increase in variable production costs. Thereby, we identify limited capacity as a potential driver for a DSR. In the literature, the DSR is mainly considered as a strategy to mitigate risks. On the contrary, we show that a switch from a DSR to an SSR can be optimal in response to limited capacity if the increase in variable production costs exceeds the increase in capacity consumption, to avoid cannibalization of the new generation by the old generation.

We find that for new product generation with a medium increase in quality, the selection of rollover strategies is nonmonotone in the available capacity. This implies that a change in the rollover strategy that is optimal to mitigate a certain limiting capacity has to be reversed in case the capacity shortages are more severe.

We examined the profit losses that occur if a capacity constraint is ignored when deciding on the sales rollover strategy and pricing. These losses compared to an integrated sales and operations planning approach can be substantial and increase with the severity of capacity shortages and the quality increase of the new generation. In conclusion, our results show that differentiating sales and production rollover strategies is an effective way to mitigate the effects of a limited capacity.

The proposed model leaves several avenues for future research. For example, instead of allowing the difference in quality between product generations and operational parameters such as the increase in variable production costs and capacity consumption to be varied independently, a positive correlation could be imposed. In addition, an endogenous decision regarding a capacity exten- 
sion, e.g., via overtime can also be considered. While the presented model assumes no stockout-based substitution, we analyzed a model variant with stockout-based substitution in which the available products are consumed according to a fluid model. The numerical results indicate that for optimal decisions stockoutbased substitution never occurs. How this result extends to a setting with more a sophisticated model of stockout-based substitutions is another direction for future research. Moreover, stochastic demand and stochastic production processes, and limited flexibility in pricing due to competition are also worth investigating.

\section{Acknowledgements}

Research leading to these results has received funding from the EU ECSEL Joint Undertaking under Grant Agreement No. 737459 (Project Productive 4.0), from TÜBITAK (217M145), and from the German Federal Ministry of Education and Research BMBF (FKZ16ESE0189). The first author was in part supported by the Julius Paul Stiegler Memorial Foundation.

\section{Appendix A. Derivation of the demand function}

Let $F$ be the cumulative density function of a uniform distribution between 0 and 1 . Further, the demand potential is normalized to $d=1$. If the old and the new generations are sold simultaneously, the demand for the old generation $d_{n, o}^{o}$ is given by the share of customers that have a quality level valuation of $\theta<\theta^{\prime}$ and $u^{o}(\theta)>0$, i.e., $\theta>\frac{p^{o}}{q}$,

$$
\begin{aligned}
d_{n, o}^{o} & =\left(F\left(\theta^{\prime}\right)-F\left(\min \left\{\frac{P^{o}}{q} ; \theta^{\prime}\right\}\right)\right) d \\
& =\min \left\{1 ; \theta^{\prime}\right\}-\min \left\{1 ; \frac{P^{o}}{q} ; \theta^{\prime}\right\} .
\end{aligned}
$$

In the case that only the old product is sold, all customers with a positive net utility will buy the old product and the demand $d_{o}^{o}$ is

$d_{o}^{o}=\left(1-F\left(\frac{P^{o}}{q}\right)\right) d=1-\min \left\{1 ; \frac{P^{o}}{q}\right\}$.

Analogously, the demand for the new product when both product generations are sold $d_{n, o}^{n}$, is given by the share of customers with a positive net utility for the new product $u^{n}(\theta)>0$, i.e., $\theta>\frac{P^{n}}{q(1+\Delta)}$ and a quality valuation higher than the indifference point $\theta>\theta^{\prime}$

$$
\begin{aligned}
d_{n, o}^{n} & =\left(1-F\left(\max \left\{\frac{P^{n}}{q(1+\Delta)} ; \theta^{\prime}\right\}\right)\right) d \\
& =1-\min \left\{1 ; \max \left\{\frac{P^{n}}{q(1+\Delta)} ; \theta^{\prime}\right\}\right\} .
\end{aligned}
$$

Similarly, all the customers with a positive net utility buy if only the new generation is sold, resulting in demand $d_{n}^{n}$ with

$d_{n}^{n}=\left(1-F\left(\frac{P^{n}}{q(1+\Delta)}\right) d=1-\min \left\{1 ; \frac{P^{n}}{q(1+\Delta)}\right\}\right.$.

In the following, we normalize the base quality level of the old generation to $q=1$ so that (9) and (10) lead to Constraint (2f) on the old product and (11) and (12) lead to the constraint on the sales of the new product $(2 \mathrm{~g})$.

\section{Appendix B. Proofs}

Lemma 1. All optimal solutions to Problem (2) can be found in the subspace given by $0 \leq P^{o} \leq 1,(\Delta+1) P^{o} \leq P^{n} \leq P^{o}+\Delta$, $S^{o} \leq \theta^{\prime}-$ $P^{o}$, and $S^{n} \leq 1-\theta^{\prime}$.
Proof of Lemma 1. The net utility is $u^{0}<0$ for $P^{0}>1$ and $u^{n}<0$ for $P^{n}>1+\Delta$, respectively. Hence, the demand for old and new products is 0 , respectively and according to (2a) changes in the prices do not change the objective value. It has to be shown that by excluding $0 \leq(\Delta+1) P^{o}<P^{n}$ and $P^{n}>P^{o}+\Delta \geq \Delta+1$ no unique optimal solution is excluded.

For $P^{n}<(\Delta+1) P^{0}$ it follows from (2f) that $S^{0}=0$, consequently decreasing $P^{o}$ to $P^{o^{\prime}}$ such that $P^{n}=P^{o^{\prime}}+\Delta$ yields the same profit. From $P^{n}>P^{o}+\Delta$ it follow that $\theta^{\prime}>1$, hence $S^{n}=0$ according to $(2 \mathrm{~g})$, consequently decreasing $P^{n}$ to $P^{n^{\prime}}$ such that $\theta^{\prime}=$ $1 \Leftrightarrow P^{n^{\prime}}=P^{0}+\Delta$ results in the same profit. Hence, a restriction to $(\Delta+1) P^{o} \leq P^{n} \leq P^{o}+\Delta$ does not lead to suboptimal solutions.

Given the price restrictions $P^{o} \leq 1$ and $(\Delta+1) P^{o} \leq P^{n} \leq P^{o}+\Delta$, the sales constraints (2f) and (2g) simplify to

$S^{o} \leq \begin{cases}\frac{P^{n}-P^{o}}{\Delta}-P^{o}=d_{n, o}^{o} & \text { if } S^{n}>0, \\ 1-P^{o}=d_{o}^{o} & \text { otherwise, }\end{cases}$

$S^{n} \leq \begin{cases}1-\frac{P^{n}-P^{o}}{\Delta}=d_{n, o}^{n} & \text { if } S^{o}>0, \\ 1-\frac{P^{n}}{1+\Delta}=d_{n}^{n} & \text { otherwise. }\end{cases}$

Next we show that for every $0<P^{0}<1$ there is a unique $(\Delta+$ 1) $P^{o} \leq P^{n} \leq \Delta+P^{o}$ for which $d_{o}^{o}=d_{n, o}^{o} \geq 0$ and $d_{n, o}^{n}=0$. From $d_{o}^{o}=$ $d_{n, o}^{o}$ it follows that $\frac{P^{n}-P^{o}}{\Delta}-P^{o}=1-P^{o} \Leftrightarrow P^{n}=\Delta+P^{o}$. From $d_{n, o}^{n}=$ 0 it follows $1-\frac{P^{n}-P^{o}}{\Delta}=0 \Leftrightarrow P^{n}=\Delta+P^{o}$. Hence, it is sufficient to consider $S^{o} \leq \frac{P^{n}-P^{o}}{\Delta}-P^{o}$.

Conversely, for every $(\Delta+1) P^{0} \leq P^{n} \leq 1+P^{o}$ there is a unique $0 \leq P^{o} \leq 1$ for which $d_{n}^{n}=d_{n, o}^{n} \geq 0$ and $\bar{d}_{n, o}^{o}=0$. From $d_{n}^{n}=d_{n, o}^{n}$ it follows $1-\frac{P^{n}-P^{o}}{\Delta}=1-\frac{P^{n}}{1+\Delta} \Leftrightarrow P^{o}=\frac{P^{n}}{1+\Delta}$ and from $d_{n, o}^{o}=0$ it follows $P^{o}=\frac{P^{n}-P^{o}}{\Delta}-P^{o} \Leftrightarrow P^{o}=\frac{P^{n}}{1+\Delta}$. Hence, it is sufficient to consider $S^{n} \leq 1-\frac{P^{n}-P^{o}}{\Delta}$.

Lemma 2. All optimal solutions to Problem (2) fulfill Constraints (2f) and $(2 g)$ with equality.

Proof of Lemma 2. Suppose an optimal solution does not fulfill Constraint (2f) with equality. By increasing $P^{o}$ to $P^{0^{\prime}}$ such that Constraint (2f) is binding, the objective value can be improved. The slack in Constraint $(2 \mathrm{~g})$ is non-decreasing in $P^{0}$ and all other constraints remain unaffected. Hence, the solution with $P^{0^{\prime}}$ is feasible but has a higher objective value which contradicts the assumption that the original solution is an optimal solution. Analogous arguments hold for non-binding Constraint $(2 \mathrm{~g})$ and increases in $P^{n}$.

Lemma 3. The optimal decisions $P^{0, *}, X^{0, *}, S^{0, *}, P^{n, *}, X^{n, *}=S^{n, *}$ and the resulting profit $\pi_{\infty}^{*}$ for Problem (2) with $k=k^{0}=\infty$ are:

\begin{tabular}{llll}
\hline & $\Delta \leq \alpha c$ & $\alpha c<\Delta<\alpha$ & $\alpha \leq \Delta$ \\
\hline$P_{\infty}^{0, *}$ & $\frac{c+1}{2}$ & $\frac{c+1}{2}$ & - \\
$P_{\infty}^{n, *}$ & - & $\frac{c(1+\alpha)+1+\Delta}{2}$ & $\frac{c(1+\alpha)+1+\Delta}{2}$ \\
$S_{\infty}^{0, *}=X_{\infty}^{0, *}$ & $\frac{1-c}{2}$ & $\frac{c(\alpha-\Delta)}{2 \Delta}$ & 0 \\
$S_{\infty}^{n, *}=X_{\infty}^{n, *}$ & 0 & $\frac{\Delta-\alpha c}{2 \Delta}$ & $\frac{1+\Delta-c(1+\alpha)}{2(1+\Delta)}$ \\
$\pi_{\infty}^{*}$ & $\frac{(c-1)^{2}}{4}$ & $\frac{c^{2} \Delta+\alpha^{2} c^{2}-2 \alpha c \Delta-2 c \Delta+\Delta^{2}+\Delta}{4 \Delta}$ & $\frac{\frac{(c(1+\alpha)-(1+\Delta))^{2}}{4(1+\Delta)}}{4}$ \\
\hline
\end{tabular}

Proof of Lemma 3. Due to the unlimited capacity and nonnegative holding cost, pre-production is never optimal and $X_{\text {pre, }}^{0, *}=0$ and production is always aligned with sales, i.e., $S^{o, *}=X^{o, *}$ and $S^{n, *}=$ $X^{n, *}$. With Lemma 1 and 2, Problem (2) further simplifies to

$\max _{P^{n}, P^{o}} \pi_{\infty}=\left(P^{n}-c(1+\alpha)\right)\left(1-\frac{P^{n}-P^{o}}{\Delta}\right)+\left(P^{o}-c\right)\left(\frac{P^{n}-P^{o}}{\Delta}-P^{o}\right)$ 
s.t.

$$
\frac{P^{n}-P^{o}}{\Delta}-P^{o} \geq 0
$$

$1-\frac{P^{n}-P^{o}}{\Delta} \geq 0$

$0 \leq P^{o} \leq 1,(\Delta+1) P^{o} \leq P^{n} \leq \Delta+P^{o}$.

The necessary condition for optimal prices are obtained by equating the partial derivatives to 0

$\frac{\partial \pi_{\infty}}{\partial P^{o}}=\frac{c(\Delta-\alpha)+2\left(P^{n}-P^{o}(1+\Delta)\right)}{\Delta}=0 \Leftrightarrow P^{o}=\frac{2 P^{n}+c(\Delta-\alpha)}{2(1+\Delta)}$,

$\frac{\partial \pi_{\infty}}{\partial P^{n}}=\frac{\alpha c+\Delta+2\left(P^{o}-P^{n}\right)}{\Delta}=0 \Leftrightarrow P^{o}=\frac{2 P^{n}-\Delta-\alpha c}{2}$.

with the resulting prices

$P_{\infty}^{n, *}=\frac{c(1+\alpha)+1+\Delta}{2}>0$,

$P_{\infty}^{o, *}=\frac{c+1}{2}>0$

The obtained prices mark a global maximum as the Hessian matrix

$H_{\pi_{\infty}}=\left(\begin{array}{cc}-\frac{2(1+\Delta)}{\Delta} & \frac{2}{\Delta} \\ \frac{2}{\Delta} & -\frac{2}{\Delta}\end{array}\right)$,

is negative definite because it is symmetric, $-\frac{2(1+\Delta)}{\Delta}<0$, and $\left|H_{\pi_{\infty}}\right|=\frac{4}{\Delta}>0$. The resulting optimal sales and profit are

$$
\begin{aligned}
S_{\infty}^{n, *}= & \frac{\Delta-\alpha c}{2 \Delta}, S_{\infty}^{o, *}=\frac{c(\alpha-\Delta)}{2 \Delta}, \\
& \pi_{\infty}^{*}=\frac{c^{2} \Delta+\alpha^{2} c^{2}-2 \alpha c \Delta-2 c \Delta+\Delta^{2}+\Delta}{4 \Delta} .
\end{aligned}
$$

We find the conditions for a DSR and DPR from the conditions for positive sales of both generations

$S_{\infty}^{n, *}>0 \Leftrightarrow \alpha c<\Delta$ and $S_{\infty}^{o, *}>0 \Leftrightarrow \alpha>\Delta$.

Consequently, for $\tilde{\Delta}_{1}=\alpha \leq \Delta$ an SSR is optimal, for which $\frac{P^{n, *}-P^{0}}{\Delta}-P^{o}=0 \Leftrightarrow P^{o, *}=\frac{1}{2}\left(1+\frac{c(1+\alpha)}{1+\Delta}\right)$. The optimal sales of both generations and the resulting profit are

$$
\begin{gathered}
S_{\infty}^{o, *}=0, S_{\infty}^{n, *}=\frac{1+\Delta-c(1+\alpha)}{2(1+\Delta)}>0, \\
\pi_{\infty}^{*}=\frac{(c(1+\alpha)-(1+\Delta))^{2}}{4(1+\Delta)} .
\end{gathered}
$$

The non-negativity of the sales condition is fulfilled for all considered cases $c(1+\alpha)<(1+\Delta)$. Analogously, for $\tilde{\Delta}_{2}=c \alpha \geq \Delta$ a "do not introduce" strategy is optimal, where the optimal decisions follow from $1-\frac{P^{n}-P^{o, *}}{\Delta}=0 \Leftrightarrow P^{n, *}=\frac{1}{2}(1+c+\Delta(1-c))$,

$S_{\infty}^{o, *}=\frac{1-c}{2}>0, S_{\infty}^{n, *}=0, \pi_{\infty}^{*}=\frac{(c-1)^{2}}{4}$.

The non-negativity of the sales condition is fulfilled for all considered cases $1 \geq c$

Proof of Corollary 1. The value of $\bar{k}$ follows immediately from $X_{\infty}^{0, *}+(1+\beta) X_{\infty}^{n, *}=\bar{k}$ with $X_{\infty}^{o, *}, X_{\infty}^{n, *}$ according to Lemma 3 .

Proof of Theorem 1. From Corollary 1 , we find the capacity $\bar{k}$ such that capacity becomes binding (3). Next, we analyze the optimal decisions for $k<\bar{k}$ and relaxed Constraint (2d). With Lemma 1 and 2 , we find the sales of the old and the new generation

$S^{o}=\left(\frac{P^{n}-P^{o}}{\Delta}-P^{o}\right)=X^{o}+X_{p r e}^{o}, S^{n}=\left(1-\frac{P^{n}-P^{o}}{\Delta}\right)=X^{n}$.

This leads to the following optimization problem

$$
\begin{aligned}
\max _{P^{o}, P^{n}, X_{p r e}^{o}} \pi_{k}= & \left(P^{n}-c(1+\alpha)\right)\left(1-\frac{P^{n}-P^{o}}{\Delta}\right) \\
& +\left(P^{o}-c\right)\left(\frac{P^{n}-P^{o}}{\Delta}-P^{o}\right)-h X_{\text {pre }}^{o}
\end{aligned}
$$

s.t.

$$
\begin{aligned}
& \left(\frac{P^{n}-P^{o}}{\Delta}-P^{o}\right)-X_{\text {pre }}^{o}+(1+\beta)\left(1-\frac{P^{n}-P^{o}}{\Delta}\right)=k, \\
& \frac{P^{n}-P^{o}}{\Delta}-P^{o} \geq X_{\text {pre }}^{o}, \\
& 1-\frac{P^{n}-P^{o}}{\Delta} \geq 0, \\
& 0 \leq P^{o} \leq 1,(\Delta+1) P^{o} \leq P^{n} \leq \Delta+P^{o}, X_{\text {pre }}^{o} \geq 0 .
\end{aligned}
$$

From Constraint (26b), we obtain

$P^{n}=\frac{\Delta\left(\beta-X_{p r e}^{o}-k-P^{o}+1\right)}{\beta}+P^{o} \quad \beta>0$,

$P^{o}=1-X_{\text {pre }}^{o}-k \quad \beta=0$.

Hence, we split the analysis in the cases $\beta>0$ and $\beta=0$. We start with the case $\beta>0$ and fix the decision variable $X_{\text {pre }}^{o}$ to $x_{\text {pre }}^{o}$. The resulting optimization problem is given by

$\max _{P_{0}} \pi_{k}$

$=\frac{\beta(\Delta-\alpha c)\left(x_{p r e}^{o}+k+P^{o}-1\right)-\beta^{2}\left(P^{o}-(c+1) P^{o}+c+h x_{p r e}^{o}\right)-\Delta\left(x_{p r e}^{o}+k+P^{o}-1\right)^{2}}{\beta^{2}}$

s.t.

$$
\begin{aligned}
& P^{o} \geq 1-x_{\text {pre }}^{o}-k, \\
& P^{o} \leq 1-x_{\text {pre }}^{o}-\frac{k}{1+\beta} .
\end{aligned}
$$

We split the analysis in the unconstrained and two constrained cases. For the unconstrained problem, the first and second partial derivative with respect to $P^{0}$ of the profit are

$\frac{\partial \pi_{k}}{\partial P^{o}}=\frac{\Delta\left(\beta-2\left(x_{p r e}^{o}+k+P^{o}-1\right)\right)}{\beta^{2}}-\frac{\alpha c}{\beta}+c-2 P^{o}+1$,

$\frac{\partial^{2} \pi_{k}}{\partial P^{o^{2}}}=-\frac{2 \Delta}{\beta^{2}}-2<0$.

We find the price $P_{k}^{*, o}$ for the unconstrained case from the following necessary condition for extreme points

$\frac{\partial \pi_{k}}{\partial P^{o}}=0 \Leftrightarrow P_{k}^{*, o}=\frac{\beta^{2}(c+1)+\beta(\Delta-\alpha c)-2 \Delta\left(x_{p r e}^{o}+k-1\right)}{2\left(\beta^{2}+\Delta\right)}$.

Due to the concavity of $\pi_{k}$ in $P^{o}\left(\frac{\partial^{2} \pi_{k}}{\partial P^{2}}<0\right)$, we find the following two optimal values in case the Constraints (29b) or (29c) become binding:

$P_{\underline{k}}^{*, o}=1-x_{\text {pre }}^{o}-k$, 
$P_{\bar{k}}^{*, o}=1-x_{\text {pre }}^{o}-\frac{k}{1+\beta}$.

Which of the constraints is binding depends on the value of $x_{\text {pre }}^{o}$. We identify the following properties of the optimal unconstrained, and constrained prices with respect to the amount of preproduction

$\frac{\partial P_{k}^{*, o}}{\partial x_{\text {pre }}^{o}}=-\frac{\Delta}{\Delta+\beta^{2}}, \quad \frac{\partial^{2} P_{k}^{*, n}}{\partial x_{p r e}^{o}}=0$,

$\frac{\partial P_{\bar{k}}^{*, o}}{\partial x_{p r e}^{o}}=-1, \quad \frac{\partial^{2} P_{\bar{k}}^{*, o}}{\partial x_{p r e}^{o}{ }^{2}}=0$,

$\frac{\partial P_{\underline{k}}^{*, o}}{\partial x_{\text {pre }}^{o}}=-1, \quad \frac{\partial^{2} P_{\underline{k}}^{*, o}}{\partial x_{p r e}^{o}}=0$.

Hence, the unique value of $x_{\text {pre }}^{o}$ at which $P_{\bar{k}}^{*, o}=P_{k}^{*, o}$ is given by

$x_{\text {pre }, \bar{k}}^{o}=$

$\frac{-\beta^{2}(c-1)-\beta(c+\Delta+2 k-1)+\Delta(2 k-1)+\alpha(c(1+\beta))}{2 \beta(1+\beta)}$

and the value $x_{\text {pre }}^{o}$ that ensures $P_{k}^{*, o}$ is equal to $P_{\underline{k}}^{*, o}$ is obtained at

$x_{\text {pre } \underline{\underline{k}}}^{o}=\frac{\beta-\Delta-c(\beta-\alpha)}{2 \beta}-k$.

Note that $x_{\text {pre }, \bar{k}}^{o} \geq x_{\text {pre }, \underline{k}}^{o}$ and the partial derivative of the profit with respect to the pre-production is continuous at (38) and (39), i.e.,

$$
\begin{aligned}
& \frac{\partial \pi_{k}\left(P_{\underline{k}}^{*, o}, x_{p r e}^{o}=x_{p r e, \underline{k}}^{o}\right)}{\partial x_{p r e}^{o}}=\frac{\partial \pi_{k}\left(P_{k}^{*, o}, x_{p r e}^{o}=x_{p r e, \underline{k}}^{o}\right)}{\partial x_{p r e}^{o}} \\
& \text { and } \frac{\partial \pi_{k}\left(P_{\bar{k}}^{*, o}, x_{p r e}^{o}=x_{p r e, \bar{k}}^{o}\right)}{\partial x_{p r e}^{o}}=\frac{\partial \pi_{k}\left(P_{k}^{*, o}, x_{p r e}^{o}=x_{p r e, \bar{k}}^{o}\right)}{\partial x_{p r e}^{o}},
\end{aligned}
$$

and decreasing in $x_{\text {pre }}^{o}$ because

$$
\begin{aligned}
& \frac{\partial \pi_{k}\left(P_{k}^{*, o}\right)}{\partial x_{\text {pre }}^{o}}=\frac{\Delta\left(1+\beta-c-h-2\left(x_{\text {pre }}^{o}+k\right)\right)-\beta(h \beta+c \alpha)}{\beta^{2}+\Delta}, \\
& \frac{\partial^{2} \pi_{k}\left(P_{k}^{*, o}\right)}{\partial x_{\text {pre }}^{o}}=\frac{-2 \Delta}{\beta^{2}+\Delta}<0,
\end{aligned}
$$

$\frac{\partial \pi_{k}\left(P_{\bar{k}}^{*, o}\right)}{\partial x_{\text {pre }}^{o}}=1-2\left(x_{\text {pre }}^{o}+\frac{k}{1+\beta}\right)-c-h, \quad \frac{\partial^{2} \pi_{k}\left(P_{\bar{k}}^{*, o}\right)}{\partial x_{\text {pre }}^{o}{ }^{2}}=-2<0$,

$\frac{\partial \pi_{k}\left(P_{\underline{k}}^{*, o}\right)}{\partial x_{\text {pre }}^{o}}=1-2\left(x_{\text {pre }}^{o}+k\right)-c-h, \quad \frac{\partial^{2} \pi_{k}\left(P_{\underline{k}}^{*, o}\right)}{\partial x_{\text {pre }}^{o}}=-2<0$.

Hence, three cases can be differentiated based on whether the intersections $x_{p r e, \bar{k}}^{0}$ and $x_{p r e, \underline{k}}^{0}$ occur for $x_{p r e}^{0} \leq 0$ or $x_{p r e}^{0}>0$ because the optimal prices are all linear in $x_{\text {pre }}^{o}$ (see (35)-(37)).

Case $1\left(x_{p r e, \bar{k}}^{o} \leq 0 \wedge x_{p r e, \underline{k}}^{o}<0 \wedge \beta>0\right)$ :

From $x_{p r e, \bar{k}}^{o} \leq 0$ it follows,

$$
\begin{aligned}
k \leq\left(1+\frac{c(\beta-\alpha)}{(\Delta-\beta)}\right) \frac{(\beta+1)}{2}=\hat{k}_{1} & \text { for } \Delta>\beta, \\
k \geq\left(1+\frac{c(\beta-\alpha)}{(\Delta-\beta)}\right) \frac{(\beta+1)}{2}=\hat{k}_{1} & \text { for } \Delta<\beta, \\
\Delta \geq \alpha=\tilde{\Delta}_{1} & \text { for } \Delta=\beta .
\end{aligned}
$$

From $x_{p r e, \underline{k}}^{o}<0$ it follows,

$k>\frac{\beta-\Delta-c(\beta-\alpha)}{2 \beta}=\hat{k}_{2}$.

For this case Constraint (29c) is binding. Dependent on the partial derivative of the profit with respect to the pre-production, two subcases have to be differentiated.

Case 1.1: No pre-production, $X_{\text {pre }}^{0, *}=0$, is optimal for $\frac{\partial \pi_{k}\left(P_{\vec{k}}^{*, o}, x_{\text {pre }}^{o}=0\right)}{\partial x_{\text {pre }}^{o}} \leq 0$, i.e.,

$k \geq \frac{(\beta+1)(1-c-h)}{2}=\tilde{k}_{1}$

By substituting $X_{\text {pre }}^{0, *}$ in (34), (27), and (25), we find

$$
\begin{aligned}
& P_{k}^{o, *}=1-\frac{k}{1+\beta}, P^{n, *}=\frac{(1+\Delta)(1+\beta-k)}{(1+\beta)}, \\
& S_{k}^{0, *}=X_{k}^{o, *}=0, \quad S_{k}^{n, *}=X_{k}^{n, *}=\frac{k}{\beta+1},
\end{aligned}
$$

and from (29a) the resulting profit

$\pi_{k}^{*}=\frac{k((\beta+1)(\Delta+1-c(\alpha+1))-(\Delta+1) k)}{(\beta+1)^{2}}$.

Case 1.2: Pre-production is optimal for $\frac{\partial \pi_{k}\left(P_{\vec{k}}^{*, o}, x_{\text {pre }}^{o}=0\right)}{\partial x_{\text {pre }}^{o}}>0$, i.e.,

$k<\frac{(\beta+1)(1-c-h)}{2}=\tilde{k}_{1}$.

The optimal pre-production $X_{\text {pre }}^{o, *}$ is derived from

$$
\begin{aligned}
& \frac{\partial \pi_{k}\left(P_{\bar{k}}^{*, o}\right)}{\partial x_{\text {pre }}^{o}}=1-2\left(x_{\text {pre }}^{o}+\frac{k}{1+\beta}\right)-c-h=0 \\
& \Leftrightarrow X_{\text {pre }}^{o, *}=\frac{1-c-h}{2}-\frac{k}{1+\beta} .
\end{aligned}
$$

By substituting $X_{\text {pre }}^{o, *}$ in (34), (27), (25), and (29a) we find

$P_{k}^{o, *}=\frac{c+h+1}{2}, P_{k}^{n, *}=\frac{c+h+1}{2}+\Delta\left(1-\frac{k}{(1+\beta)}\right), X_{k}^{o, *}=0$,

$S_{k}^{0, *}=X_{\text {pre }}^{0, *}=\frac{1-c-h}{2}-\frac{k}{1+\beta}, S_{k}^{n, *}=X_{k}^{n, *}=\frac{k}{\beta+1}$,

$\pi_{k}^{*}=$ $\frac{(1+\beta)\left(\beta(c+h-1)^{2}+c^{2}+2 c(h-2 \alpha k-1)+4 h k+(h-1)^{2}\right)+4 \Delta k(\beta-k+1)}{4(\beta+1)^{2}}$.

Case $2\left(x_{p r e, \bar{k}}^{o}>0 \wedge x_{p r e, \underline{k}}^{o}<0 \wedge \beta>0\right)$ :

In addition to Condition (45), from $x_{p r e, \bar{k}}^{o}>0$ it follows

$k>\left(1+\frac{c(\beta-\alpha)}{(\Delta-\beta)}\right) \frac{(\beta+1)}{2}=\hat{k}_{1}$ for $\Delta>\beta$,

$k<\left(1+\frac{c(\beta-\alpha)}{(\Delta-\beta)}\right) \frac{(\beta+1)}{2}=\hat{k}_{1}$ for $\Delta<\beta$,

$\Delta<\alpha=\tilde{\Delta}_{1}$ for $\Delta=\beta$.

Depending on the partial derivative of the profit with respect to the pre-production, three subcases have to be differentiated.

Case 2.1: No pre-production, $X_{\text {pre }}^{o, *}=0$, is optimal for $\frac{\partial \pi_{k}\left(P_{k}^{*, 0}, x_{p r e}^{o}=0\right)}{\partial x_{\text {pre }}^{0}} \leq 0$, i.e.,

$k \geq \frac{\Delta(\beta+1)-c(\alpha \beta+\Delta)-h\left(\Delta+\beta^{2}\right)}{2 \Delta}=\tilde{k}_{2}$.

In this case Problem (29) is unconstrained. By substituting $X_{\text {pre }}^{0, *}=0$ in (32), (27), and (25) we find 
$P_{k}^{o, *}=\frac{\beta(-\alpha c+\beta(c+1))+\Delta(2(1-k)+\beta)}{2\left(\Delta+\beta^{2}\right)}$,

$P_{k}^{n, *}=$

$\frac{\Delta^{2}+\beta(-\alpha c+\beta(c+1))+\Delta\left(-2(1+\beta) k+\left(c(\alpha-\beta)+2\left(1+\beta+\beta^{2}\right)\right)\right)}{2\left(\Delta+\beta^{2}\right)}$

$S_{k}^{o, *}=X_{k}^{o, *}=\frac{(1+\beta)(c(\alpha-\beta)+(\beta-\Delta))+2 k(\Delta-\beta)}{2\left(\Delta+\beta^{2}\right)}$,

$S_{k}^{n, *}=X_{k}^{n, *}=\frac{2 \beta k+(c(\beta-\alpha)+\Delta-\beta)}{2\left(\Delta+\beta^{2}\right)}$,

and from (29a) the resulting profit

$\pi_{k}^{*}=$

$\underline{\beta^{2}(c-1)^{2}+\Delta^{2}-4 \Delta k(c-1+k)-2 c \Delta \alpha+c^{2} \alpha^{2}+2 \beta(c-1+2 k)(\Delta-c \alpha)}$ $4\left(\Delta+\beta^{2}\right)$

Case 2.2: Pre-production is optimal for the case $\frac{\partial \pi_{k}\left(P_{k}^{*, o}, x_{\text {pre }}^{o}=0\right)}{\partial x_{\text {pre }}^{o}}>0$, i.e.,

$k<\frac{\Delta(\beta+1)-c(\alpha \beta+\Delta)-h\left(\Delta+\beta^{2}\right)}{2 \Delta}=\tilde{k}_{2}$

and $\frac{\partial \pi_{k}\left(P_{k}^{*, o}, x_{p r e}^{o}=x_{p r e, \bar{k}}^{o}\right)}{\partial x_{p r e}^{o}} \leq 0$, i.e.,

$k \geq \frac{(\beta+1)(\Delta-\beta h-\alpha c)}{2 \Delta}=\tilde{k}_{3}$.

In this case Problem (29) is unconstrained. The optimal value of pre-production is determined from

$\frac{\partial \pi_{k}\left(P_{k}^{*, o}\right)}{\partial x_{\text {pre }}^{o}}=0 \Leftrightarrow X_{\text {pre }}^{o, *}=\frac{\Delta(1+\beta)-c(\Delta+\alpha \beta)-h\left(\Delta+\beta^{2}\right)}{2 \Delta}-k$.

By substituting $X_{\text {pre }}^{0, *}$ in (32), (27), and (25), we find

$P_{k}^{n, *}=\frac{1+\Delta+c(1+\alpha)+h(1+\beta)}{2}, P_{k}^{o, *}=\frac{1+c+h}{2}$,

$X_{k}^{o, *}=\frac{(1+\beta)(c \alpha+h \beta)+\Delta(2 k-(\beta+1))}{2 \Delta}$,

$S_{k}^{o, *}=\frac{c(\alpha-\Delta)+h(\beta-\Delta)}{2 \Delta}, S_{k}^{n, *}=X_{k}^{n, *}=\frac{\Delta-\alpha c-h \beta}{2 \Delta}$,

and from (29a) the resulting profit

$\pi_{k}^{*}=$

$\frac{\Delta^{2}+\Delta\left(1+c^{2}+h(-2-2 \beta+h+4 k)+2 c(-1+h-\alpha)\right)+(\beta h+c \alpha)^{2}}{4 \Delta}$.

Case 2.3: For the case $\frac{\partial \pi_{k}\left(P_{k}^{*, o}, x_{p r e}^{o}=x_{p r e, \bar{k}}^{o}\right)}{\partial x_{p r e}^{o}}>0$, i.e.,

$k<\frac{(\beta+1)(\Delta-\beta h-\alpha c)}{2 \Delta}=\tilde{k}_{3}$,

the optimal decisions are equivalent to Case 1.2 and given by (51).

Case $3\left(x_{\text {pre }, \bar{k}}^{o}>0 \wedge x_{p r e, \underline{k}}^{o} \geq 0 \wedge \beta>0\right)$

In addition to Condition (52), from $x_{\text {pre }, \underline{k}}^{o} \geq 0$ it follows

$k \leq \frac{\beta-\Delta-c(\beta-\alpha)}{2 \beta}=\hat{k}_{2}$.

Depending on the partial derivative of the profit with respect to the pre-production, four subcases have to be differentiated.

Case 3.1: No pre-production, $X_{\text {pre }}^{o, *}=0$, is optimal for $\frac{\partial \pi_{k}\left(P_{k}^{*, o}, x_{p r e}^{o}=0\right)}{\partial x_{p r e}^{o}} \leq 0$, i.e.,

$k \geq \frac{1-c-h}{2}=\tilde{k}_{4}$
In this case Problem (29) is constrained by Constraint (29b). By substituting $X_{\text {pre }}^{0, *}$ in (33), (27), (25), and (29a) we find

$P_{k}^{o, *}=1-k, P_{k}^{n, *}=1+\Delta-k, X_{k}^{o, *}=S_{k}^{o, *}=k$,

$X_{\text {pre }}^{o, *}=S_{k}^{n, *}=X_{k}^{n, *}=0, \pi_{k}^{*}=k(1-c-k)$.

Case 3.2: Pre-production is optimal for the case of $\frac{\partial \pi_{k}\left(P_{k}^{*, o}, x_{p r e}^{o}=0\right)}{\partial x_{p r e}^{o}}>0$, i.e.,

$k<\frac{1-c-h}{2}=\tilde{k}_{4}$,

and $\frac{\partial \pi_{k}\left(P_{k}^{*, o}, x_{p r e}^{o}=x_{p r e, \underline{k}}^{o}\right)}{\partial x_{p r e}^{0}} \leq 0$, i.e.,

$\Delta \leq \beta h+\alpha c=\tilde{\Delta}_{3}$.

In this case also Constraint (29b) is binding. The optimal value of pre-production is determined from

$\frac{\partial \pi_{k}\left(P_{\underline{k}}^{*, o}\right)}{\partial x_{\text {pre }}^{o}}=0 \Leftrightarrow X_{\text {pre }}^{o, *}=\frac{1-c-h}{2}-k$.

By substituting $X_{\text {pre }}^{0, *}$ in (33), (27), and (25), we find

$$
\begin{aligned}
& P_{k}^{o, *}=\frac{c+h+1}{2}, P_{k}^{n, *}=\frac{c+2 \Delta+h+1}{2}, X_{k}^{o, *}=k, \\
& S_{k}^{o, *}=\frac{1-c-h}{2}, S_{k}^{n, *}=X_{k}^{n, *}=0,
\end{aligned}
$$

with resulting profit

$\pi_{k}^{*}=\frac{(c+h-1)(1+3 h-c)}{4}+h k$.

Case 3.3: For the case of $\frac{\partial \pi_{k}\left(P_{k}^{*, o}, x_{p r e}^{o}=x_{p r e, \underline{k}}^{o}\right)}{\partial x_{p r e}^{0}}>0$, i.e.,

$\Delta>\beta h+\alpha c=\tilde{\Delta}_{3}$

and $\frac{\partial \pi_{k}\left(P_{k}^{*, n}, x_{p r e}^{o}=x_{p r e, \bar{k}}^{o}\right)}{\partial x_{p r e}^{0}} \leq 0$, i.e., Condition (57) is fulfilled, the optimal decisions are as in Case 2.2 given by (58) and (59).

Case 3.4 For the case of $\frac{\partial \pi_{k}\left(P_{k}^{*, o}, x_{p r e}^{o}=x_{p r e, \bar{k}}^{0}\right)}{\partial x_{p r e}^{0}}>0$, i.e., Condition (61) is fulfilled, the optimal decisions are equivalent to Cases 1.2 and 2.3 and given by (51).

The resulting optimization problem for $\beta=0$ and $X_{\text {pre }}^{o}$ fixed to $x_{\text {pre }}^{o}$ is given by

$$
\begin{aligned}
& \max _{P^{n}} \pi_{k}=\left(P^{n}-(\alpha+1) c\right)\left(1-\frac{x_{p r e}^{o}+k+P^{n}-1}{\Delta}\right) \\
& -\frac{\left(c+x_{p r e}^{o}+k-1\right)\left((\Delta+1)\left(x_{p r e}^{o}+k-1\right)+n\right)}{\Delta}-h x_{p r e}^{o}
\end{aligned}
$$

s.t.

$P^{n} \leq 1+\Delta-x_{\text {pre }}^{o}-k=P_{\bar{k}}^{*, n}$,

$P^{n} \geq 1+\Delta-x_{\text {pre }}^{o}-k-\Delta k=P_{\underline{k}}^{*, n}$.

Problem (71) is analyzed analogous to Problem (29). First and second order optimality conditions for the objective function (71a) lead to $P_{k}^{*, n}=\frac{1}{2}\left(\alpha c+\Delta-2 x_{p r e}^{o}-2 k+2\right)$ and results in the following three cases depending on whether one of the Constraints (71b) or (71c) is binding. The profit is concave for all three prices because

$\frac{\partial^{2} \pi_{k}\left(P_{\underline{k}}^{*, n}\right)}{\partial x_{\text {pre }}^{o}}=\frac{\partial^{2} \pi_{k}\left(P_{k}^{*, n}\right)}{\partial x_{\text {pre }}^{o}}=-2<\frac{\partial^{2} \pi_{k}\left(P_{\bar{k}}^{*, n}\right)}{\partial x_{\text {pre }}^{o}}=-1<0$.

Case $1\left(P_{\underline{k}}^{*, n} \geq P_{k}^{*, n}\right)$ : From $P_{\underline{k}}^{*, n} \geq P_{k}^{*, n}$ it follows that $k \leq \frac{\Delta-\alpha c}{2 \Delta}=$ $\hat{k}_{1}$. Whether inventory is used depends on $\frac{\partial \pi_{k}\left(P_{k}^{*, n}, x_{p r e}^{o}=0\right)}{\partial x_{p r e}^{o}}$. For 
$\frac{\partial \pi_{k}\left(P_{k}^{*, n}, x_{p r e}^{o}=0\right)}{\partial x_{p r e}^{0}} \leq 0$, i.e., Condition (46), no inventory is used and the optimal decisions are as given by (47) with resulting optimal profit (48). In contrast, for $\frac{\partial \pi_{k}\left(P_{k}^{*, n}, x_{p r e}^{o}=0\right)}{\partial x_{p r e}^{o}}>0$, i.e., Condition (49), the use of inventory is optimal and the optimal decisions and the resulting profit are given by (50) and (51).

Case $2\left(P_{k}^{*, n}<P_{k}^{*, n}<P_{\bar{k}}^{*, n}\right)$ : From $P_{\bar{k}}^{*, n}<P_{k}^{*, n}$ it follows that $k>$ $\frac{\Delta-c \alpha}{2 \Delta}=\hat{k}_{1}$ and from $P_{k}^{*, n}<P_{k}^{*, n}$ it follows that $\left(\Delta>c \alpha=\tilde{\Delta}_{2} \wedge \alpha>\right.$ $0) \vee \alpha \leq 0$. Whether inventory is used depends on $\frac{\partial \pi_{k}\left(P_{k}^{*, n}, x_{p r e}^{o}=0\right)}{\partial x_{p r e}^{0}}$. For $\frac{\partial \pi_{k}\left(P_{k}^{*, n}, x_{\text {pre }}^{o}=0\right)}{\partial x_{p r e}^{o}} \leq 0$, i.e., Condition (53), no inventory is used and the optimal decisions are as given by (54). In contrast, for $\frac{\partial \pi_{k}\left(P_{k}^{*, n}, x_{\text {pre }}^{o}=0\right)}{\partial x_{\text {pre }}}>0$, i.e., Condition (56), the use of inventory is optimal and the optimal decisions are given by (58) and (59) with resulting profit (60).

Case $3\left(P_{\bar{k}}^{*, n} \leq P_{k}^{*, n}\right)$ : From $P_{k}^{*, n} \leq P_{k}^{*, n}$ it follows that $\Delta \leq c \alpha=$ $\tilde{\Delta}_{2} \wedge \alpha>0$. Whether inventory is used depends on $\frac{\partial \pi_{k}\left(P_{\vec{k}}^{*, n}, x_{\text {pre }}^{o}=0\right)}{\partial x_{p r e}^{o}}$. For $\frac{\partial \pi_{k}\left(P_{\vec{k}}^{*, n}, x_{p r e}^{o}=0\right)}{\partial x_{p r e}^{0}} \leq 0$, i.e., Condition (63), no inventory is used and the optimal decisions are as given by (64). In contrast, for $\frac{\partial \pi_{k}\left(P_{\vec{k}}^{*, n}, x_{p r e}^{o}=0\right)}{\partial x_{p r e}^{o}}>0$, i.e., Condition (65), the use of inventory is optimal and the optimal decisions are given by (67)and (68) the resulting optimal profit (69).

The optimal decisions in Table 2 follow from the following observations:

- The unique intersection of $\tilde{k}_{4}, \tilde{\Delta}_{3}$ and $\tilde{k}_{2}$ is found at $\Delta=\beta h+$ $\alpha c=\tilde{\Delta}_{3}$ and $k=\tilde{k}_{4}$, for $\beta>0$ and $\tilde{k}_{2}=\tilde{k}_{4}$ for $\beta=0$

- $\tilde{k}_{3}=0$ is given by $\tilde{\Delta}_{3}=\beta h+\alpha c$

- The unique intersection of $\hat{k}_{1}$ and $\hat{k}_{2}$ is $k=0$ and $\Delta=\beta(1-$ c) $+c \alpha$ for which $\alpha \neq \beta \wedge \beta>0$

- The unique intersection of $\hat{k}_{1}, \tilde{k}_{1}, \tilde{k}_{2}$, and $\tilde{k}_{3}$ is $\tilde{\Delta}_{4}=\frac{h \beta+c \alpha}{c+h}$ and $k=\tilde{k}_{1}$ for $\beta>0$, and for $\beta=0 \hat{k}_{1}=\tilde{k}_{3}$ and $\tilde{k}_{1}=\tilde{k}_{2}$.

- $\tilde{\Delta}_{4}=\frac{h \beta+c \alpha}{c+h}<\beta$ for $\alpha<\beta$ and $\tilde{\Delta}_{4}=\frac{h \beta+c \alpha}{c+h}>\beta$ for $\alpha>\beta$

- A unique intersection of $\hat{k}_{1}$ and $\bar{k}_{1}$ exists at $\Delta=\alpha=\tilde{\Delta}_{1}$ and $k=\frac{(1+\beta)(1-c)}{2}$ if $\beta \neq \alpha \wedge \alpha>0$

- $\hat{k}_{1}>\bar{k}_{1}$ and $\hat{k}_{1}>\bar{k}_{2}$ for $(\alpha<\beta \wedge(\Delta<\alpha \vee \Delta>\beta)) \vee(\alpha>\beta \wedge$ $(\Delta<\beta \vee \Delta>\alpha)$

- $\hat{k}_{1}<\bar{k}_{1}$ and $\hat{k}_{1}<\bar{k}_{2}$ for $(\alpha<\Delta<\beta) \vee(\alpha>\beta \wedge \beta<\Delta<\alpha)$

- $\hat{k}_{2}>0 \Leftrightarrow \Delta<\beta-c(\beta-\alpha)$

- For $\beta>\alpha \wedge 0<\Delta<\beta-c(\beta-\alpha)$ it holds that $\hat{k}_{1}>\hat{k}_{2}$

- For $0<\Delta<\beta<\alpha$ it holds that $\hat{k}_{1}>\hat{k}_{2}$ and for $\beta<\alpha \wedge \beta<$ $\Delta<\beta-c(\beta-\alpha)$ we find $\hat{k}_{1}<0$

Next we turn to the cases in which Constraint (2d) is binding. The partial derivative of the profit with respect to the preproduction is continuous and decreasing, for both $\beta>0$ (see (40) - (43)) and also for $\beta=0$ (see (72)). It follows that $X_{\text {pre }}^{o, *}=$ $\min \left\{X_{\text {pre }}^{o, *} ; k^{0}\right\}$ because $\frac{\partial \pi_{k}\left(X_{\text {pre }}^{o, *}\right)}{\partial x_{\text {pre }}^{0}}=0$ if $X_{\text {pre }}^{o, *}>0$. Hence, for the case $k^{0} \geq X_{\text {pre }}^{0, *}$, the optimal pricing, sales, and production are given by Table 2 , for $k^{0}<X_{\text {pre }}^{0, *}$ the optimal values are determined as follows.

For $\beta>0$ the optimal price of the old product $P^{o, *}$ is obtained from (33) for $x_{p r e, \underline{k}}^{o} \geq k^{0}$, from (32) for $x_{p r e, \bar{k}}^{o} \geq k^{0}>x_{p r e, \underline{k}}^{0}$, and from (34) for $k^{0}>x_{\text {pre }, \bar{k}}^{0}$. The optimal price for the new product $P^{n, *}$ is given by (27), the optimal sales $S^{0, *}, S^{n, *}$ and production quantities $X^{0, *}, X^{n, *}$ are obtained from (25).

Analogously, for $\beta=0$ the optimal price of the new product $P^{n, *}$ follows from $P^{n}=1+\Delta-X_{\text {pre }}^{o, *}-k-\Delta k$ for $k \leq \hat{k}_{1}$ (Case 1 for $\beta=0)$, from $P_{k}^{*, n}=\frac{1}{2}\left(\alpha c+\Delta-2 X_{\text {pre }}^{o, *}-2 k+2\right)$ for $\left(\Delta>\tilde{\Delta}_{2} \wedge \alpha>\right.$ $0) \vee \alpha \leq 0) \wedge k>\hat{k}_{1}$ (Case 2 for $\beta=0$ ), and from $P^{n}=1+\Delta-$
$X_{\text {pre }}^{o, *}-k$ for $\Delta \leq \tilde{\Delta}_{2} \wedge \alpha>0$ (Case 3 for $\beta=0$ ). The optimal price of the old product $P^{0, *}$ is given by (28) and the optimal sales $S^{o, *}, S^{n, *}$ and production quantities $X^{0, *}, X^{n, *}$ are obtained from (25).

Proof of Corollary 2. From Corollary 1 it follows that $\frac{\partial P^{0, *}}{\partial k}=$ $\frac{\partial P^{n, *}}{\partial k}=0$ for $k \geq \bar{k}$. Hence, we focus on the cases with $k<\bar{k}$. According to the derivations in Theorem $1, P^{0}$ and $P^{n}$ are continuous in $k$. Thus it is sufficient to show that the partial derivatives with respect to $k$ are non-positive for $k<\bar{k}$

$$
\begin{aligned}
& \frac{\partial P_{B}^{n, *}}{\partial k}=\frac{\partial P_{J}^{n, *}}{\partial k}=-\frac{1+\Delta}{1+\beta}<0, \frac{\partial P_{C}^{n, *}}{\partial k}=\frac{\partial P_{J}^{o, *}}{\partial k}=-\frac{1}{1+\beta}<0, \\
& \frac{\partial P_{E}^{o, *}}{\partial k}=\frac{\partial P_{L}^{o, *}}{\partial k}=-\frac{\Delta}{\Delta+\beta^{2}}<0, \\
& \frac{\partial P_{E}^{n, *}}{\partial k}=\frac{\partial P_{L}^{n, *}}{\partial k}=-\frac{\Delta(1+\beta)}{\Delta+\beta^{2}}<0, \frac{\partial P_{H}^{o, *}}{\partial k}=\frac{\partial P_{H}^{o, *}}{\partial k}=\frac{\partial P_{K}^{n, *}}{\partial k}=-1, \\
& \frac{\partial P_{C}^{o, *}}{\partial k}=\frac{\partial P_{F}^{o, *}}{\partial k}=\frac{\partial P_{F}^{n, *}}{\partial k}=\frac{\partial P_{G}^{o, *}}{\partial k}=0 .
\end{aligned}
$$

Proof of Corollary 3. For $\beta=0$, it follows that $\bar{k}_{3}=\frac{1-c}{2}=\bar{k}_{2}$. Hence, a change in rollover strategies could only occur for $\Delta>\tilde{\Delta}_{2}$. For $\beta=0$ it follows $\tilde{k}_{1}=\frac{1-c-h}{2} \leq \frac{1-c}{2}=\bar{k}_{3}$. Thus optimal solutions for $\bar{k}_{3} \leq k<\bar{k}_{1}$ are are always found in region $(B)$. In region $(B)$ the SSR and SPR strategy are optimal which are equivalent to the optimal sales and production strategies for $k \geq \bar{k}$ and $\Delta>\tilde{\Delta}_{2}$.

\section{References}

Bansal, A., Uzsoy, R., \& Kempf, K. (2020). Iterative combinatorial auctions for managing product transitions in semiconductor manufacturing. IISE Transactions, 52(4), 413-431.

Bernstein, F., \& Martínez-de-Albéniz, V. (2017). Dynamic product rotation in the presence of strategic customers. Management Science, 63(7), 2092-2107.

Bilginer, Ö., \& Erhun, F. (2015). Production and sales planning in capacitated new product introductions. Production and Operations Management, 24(1), 42-53.

Billington, C., Lee, H. L., \& Tang, C. S. (1998). Successful strategies for product rollovers. MIT Sloan Management Review, 39(3), 23-30.

Clark, K. B., \& Fujimoto, T. (1991). Product development performance: Strategy, organization, and management in the world auto industry. Boston, MA: Harvard Business School Press.

Erhun, F., Gonçalves, P., \& Hopman, J. (2007). The art of managing new product transitions. MIT Sloan Management Review, 48(3), 73-80.

Ferguson, M. E., \& Koenigsberg, O. (2007). How should a firm manage deteriorating inventory? Production and Operations Management, 16(3), 306-321.

Ho, T.-H., Savin, S., \& Terwiesch, C. (2002). Managing demand and sales dynamics in new product diffusion under supply constraint. Management Science, 48(2), 187-206.

Ho, T.-H., Savin, S., \& Terwiesch, C. (2011). Note: A reply to "new product diffusion decisions under supply constraints". Management Science, 57(10), 1811-1812.

Jain, D., Mahajan, V., \& Muller, E. (1991). Innovation diffusion in the presence of supply restrictions. Marketing Science, 10(1), 83-90.

Koca, E., Souza, G. C., \& Druehl, C. T. (2010). Managing product rollovers. Decision Sciences, 41(2), 403-423.

Kumar, S., \& Swaminathan, J. M. (2003). Diffusion of innovations under supply constraints. Operations Research, 51(6).

Levinthal, D. A., \& Purohit, D. (1989). Durable goods and product obsolescence. Marketing Science, 8(1), 35-56.

Li, H., \& Graves, S. C. (2012). Pricing decisions during inter-generational product transition. Production and Operations Management, 21(1), 14-28.

Li, H., Graves, S. C., \& Huh, W. T. (2014). Optimal capacity conversion for product transitions under high service requirements. Manufacturing \& Service Operations Management, 16(1), 46-60.

Liang, C., Cakanyıldırım, M., \& Sethi, S. P. (2014). Analysis of product rollover strategies in the presence of strategic customers. Management Science, 60(4), 1033-1056.

Lim, W. S., \& Tang, C. S. (2006). Optimal product rollover strategies. European Journal of Operational Research, 174(2), 905-922.

Liu, J., Zhai, X., \& Chen, L. (2018). The interaction between product rollover strategy and pricing scheme. International Journal of Production Economics, 201, 116-135.

Liu, J., Zhai, X., \& Chen, L. (2019). Optimal pricing strategy under trade-in program in the presence of strategic consumers. Omega, 84, 1-17.

Michel, A., Attali, S., \& Bush, E. (2016). Energy efficiency of white goods in Europe: Monitoring the market with sales data. Technical Report. ADEME.

Moorthy, K. S. (1988). Product and price competition in a duopoly. Marketing Science, $7(2), 141-168$. 
Mussa, M., \& Rosen, S. (1978). Monopoly and product quality. Journal of Economic Theory, 18(2), 301-317.

Negahban, A., \& Smith, J. S. (2018). Optimal production-sales policies and entry time for successive generations of new products. International Journal of Production Economics, 199, 220-232.

Policy Studies Institute (2006). Designing policy to influence consumers: Consumer behaviour relating to the purchasing of environmentally preferable goods. Technical Report.

Sale, R. S., Mesak, H. I., \& Inman, R. A. (2017). A dynamic marketing-operations interface model of new product updates. European Journal of Operational Research, 257(1), 233-242.

Shen, W., Duenyas, I., \& Kapuscinski, R. (2011). New product diffusion decisions under supply constraints. Management Science, 57(10), 1802-1810.
Shen, W., Duenyas, I., \& Kapuscinski, R. (2014). Optimal pricing, production, and inventory for new product diffusion under supply constraints. Manufacturing $\mathcal{E}$ Service Operations Management, 16(1), 28-45.

Statista (2017). Reasons for replacing old home appliances with new appliances, in the United States, as of May 2017. https://www.statista.com/statistics/711578/ united-states-statista-survey-household-appliances-purchase-reasons/.

Wieser, H., Tröger, N., \& Hübner, R. (2015). The consumers' desired and expected product lifetimes. In Plate conference (pp. 388-393). Nottingham, UK

Wilson, L. O., \& Norton, J. A. (1989). Optimal entry timing for a product line extension. Marketing Science, 8(1), 1-17.

Zhou, E., Zhang, J., Gou, Q., \& Liang, L. (2015). A two period pricing model for new fashion style launching strategy. International Journal of Production Economics, $160,144-156$. 